\title{
A Boundary Value Problem Related to the Ginzburg-Landau Model
}

\author{
Anne Boutet de Monvel-Berthier ${ }^{1}$, Vladimir Georgescu ${ }^{1,2}$ and Radu Purice ${ }^{2}$ \\ ${ }^{1}$ Université Paris VII, Equipe de Physique Mathématique et Géométrie, C.N.R.S., Tour 45-55, \\ $5^{\circ}$-Etage, 2 Place Jussieu, F-75251 Paris Cedex 05, France \\ ${ }^{2}$ Institut de Mathématiques de l'Académie des Sciences de Roumanie, Bucarest, Roumanie
}

Received March 10, 1990

This paper is dedicated to the memory of Michel Sirrugue

\begin{abstract}
We analyze the Ginzburg-Landau equation for a superconductor in the case of a 2-dimensional model: a cylindrical conductor with a magnetic field parallel to the axis. This amounts to find the extrema of the free energy

$$
\mathscr{A}_{\kappa}=1 / 2 \int_{\Omega}\left[|(\nabla-i A) \Phi|^{2}+\left|B_{A}\right|^{2}+\kappa / 4\left(|\Phi|^{2}-1\right)^{2}\right] d x,
$$

where $\Omega$ is a bounded domain with smooth boundary in $\mathbb{R}^{2}, A=\left(A_{1}, A_{2}\right)$ the vector potential, $B_{A}=\partial_{1} A_{2}-\partial_{2} A_{1}$ the magnetic field, $\Phi$ a complex field. We describe the connected components of the maximal configuration space, i.e. of the set of all $(A, \Phi)$ with components in the Sobolev space $H^{1}(\Omega)$ and such that $|\Phi|=1$ on the boundary, modulo the action of the gauge group. In the critical case $\kappa=1$ we give a complete description of the minimal configurations in each component.
\end{abstract}

\section{Introduction}

The Ginzburg-Landau model [6] has been proposed in 1950 in order to give a phenomenological description of superconductivity. It is an experimental fact that at low temperatures and weak magnetic fields some materials get an infinite electric conductivity. This phenomenon has been described by Ginzburg and Landau with the help of a complex valued field $\Phi$ interacting with the magnetic field $B . \Phi$ and the vector potential $A$ which generates $B$ satisfy a system of nonlinear partial differential equations (see [6,12 or 13]). The physical interpretation of $\Phi$ is rather complicated and was understood only after the appearance of the microscopic theory of Bardeen, Cooper and Schrieffer. Roughly speaking, this theory considers 
superconductivity as being due to some correlations between electrons which tend to form pairs, and $|\Phi(x)|^{2}$ is proportional to the number density of these pairs.

In this paper we shall consider only superconductors invariant under translations in a certain direction and magnetic fields parallel to this direction. For this reason we shall make some simplifications in the equations which will reduce the problem to a two-dimensional one.

Let $\Omega \subset \mathbb{R}^{2 \cdot}$ be an open, bounded set with $C^{\infty}$ boundary $\Gamma$ such that $\Omega$ is locally on one side of $\Gamma$. We denote $\partial_{j}=\frac{\partial}{\partial x_{j}}, D_{j}=-i \partial_{j}(j=1,2)$ and $\nabla=\left(\partial_{1}, \partial_{2}\right)$, $D=-i \nabla$ considered as vector differential operators. The magnetic field $B$ is a real-valued function on $\Omega$ of the form $B \equiv B_{A}=\partial_{1} A_{2}-\partial_{2} A_{1}$, where $A=\left(A_{1}, A_{2}\right)$ is an $\mathbb{R}^{2}$-valued map on $\Omega$. We also introduce the vector differential operators $\nabla_{A}=\nabla-i A$ and $D_{A}=D-A=-i \nabla_{A}$. Let $*$ be the rotation in $\mathbb{R}^{2}$ with an angle $\pi / 2$ in the direct sense, i.e. $*\left(x_{1}, x_{2}\right)=\left(-x_{2}, x_{1}\right)$ (we shall use this notation for $x_{1}, x_{2} \in \mathbb{C}$ also). The Ginzburg-Landau equations (in our simplified setting) can now be written in the form:

$$
\left\{\begin{array}{l}
D_{A}^{2} \Phi=\kappa \Phi \frac{1-|\Phi|^{2}}{2} \\
* \nabla B_{A}=-\operatorname{Re}\left(\overline{D_{A} \Phi} \cdot \Phi\right)
\end{array}\right.
$$

which is a system of non-linear partial differential equations for the unknown functions $\Phi: \Omega \rightarrow \mathbb{C}$ and $A: \Omega \rightarrow \mathbb{R}^{2}$. Here $\kappa$ is a real parameter (depending on the temperature, etc.). The solutions of the system have a qualitatively different behaviour for $\kappa<1$ and $\kappa>1$ (this distinguishes between type I and type II superconductivity). Equations (1.1) are written at some fixed temperature smaller than the critical one. $\Phi$ and $\kappa$ are "reduced" physical quantities (see [13], p. 30) so that $|\Phi|=0$ in normal (i.e. non-superconducting) phase and $|\Phi|=1$ in the superconducting one. One expects $0 \leqq|\Phi| \leqq 1$ in general (this is rigorously proved in Theorem 3.2 of this paper). Finally, the free energy is by convention equal to $\kappa / 4$ in the normal phase, hence in general it is equal to (see [13]):

$$
\mathscr{A}_{\kappa}(A, \Phi)=\frac{1}{2} \int_{\Omega}\left(\left|\nabla_{A} \Phi\right|^{2}+\left|B_{A}\right|^{2}+\frac{\kappa}{4}\left(|\Phi|^{2}-1\right)^{2}\right) d x
$$

and (1.1) are the variational equations associated with the above functional. We shall consider a special class of superconductors, namely those with penetration depth equal to zero (see $[12,13]$ ), which gives $|\Phi|=1$ on $\Gamma$ as boundary condition for (1.1). One may think this is a rather strong idealisation, but this is not the case, because we consider $\Gamma$ not as the real boundary of the material but as an imaginary surface located well inside the superconducting part. Thus, the only limitation of the results we obtain is that they are not accurate to describe the behaviour near the surface of the real material. On the other hand they describe quite well the vortex (anti-vortex) structure in the bulk.

In the next chapter we shall describe the configuration space of the system. We make a distinction between the physical and the mathematical configuration space. The mathematical one is the largest space on which $\mathscr{A}_{\kappa}$ is well defined and has a rather simple mathematical description. In order to define the physical 
configuration space one must take into account the fact that different mathematical configurations may describe the same physical situation, due to the so-called "gauge-invariance" of the system. An interesting physical fact, the flux quantization (London effect), will emerge from the study of the topology of the configuration space: flux quantization is an expression of the non-connectedness of the configuration space. Remark that in the case of finite superconductors we are able to solve the problem raised by Jaffe and Taubes in the footnote on page 96 of [8]. In order to prove this result we will use an extension of the definition of the degree for mappings $S^{1} \rightarrow S^{1}$ of class $H^{1 / 2}$. This is described in the appendix, which is due to L. Boutet de Monvel and, for one crucial idea, to O. Gabber, and announced in a lecture by L. Boutet de Monvel at the French-Israeli mathematical meeting in Jerusalem (April 1984). We thank them for communicating their proof to us which we present in an appendix of this paper.

In the third chapter we study the stationary points of the functional (1.2) defined on the mathematical configuration space: we prove their smoothness (modulo gauge-equivalence) and find some relations between the fields $\Phi$ and $B$ corresponding to a stationary configuration which in physical terms can be interpreted as the Meissner effect.

The case $\kappa=1$ is more thoroughly studied in Chap. 4 where we completely classify the absolute minima of the restriction of $\mathscr{A}_{1}$ to the connected components of the configuration space. In [2] Bogomol'nyi pointed out that if $\kappa=1$ then the solutions of (1.1) may be found by solving two systems of first-order differential equations. The methods we use are similar to those introduced by Taubes (see Jaffe and Taubes [8] for a complete account). We would also like to point out that the results in Chap. 2 and in the appendix of this paper are relevant in connection with conjectures 1 and 2, p. 34 of [8].

Finally, we would like to stress the fact that the boundary value problem we study is interesting due to the invariance of the functional $\mathscr{A}_{\kappa}$ under a very large group (the gauge group is infinite dimensional), which causes difficulties in the proof of the regularity of the solution especially at the boundary $\Gamma$ (the uniqueness is, of course, lost). Let us mention that the main results of this paper have been announced in our note [16].

\section{Configuration Space of the System and Flux Quantization}

We recall that the physical system under consideration is a complex field $\Phi: \Omega \rightarrow \mathbb{C}$ $\left(\Omega \subset \mathbb{R}^{2}\right.$ as in the introduction) interacting with a magnetic field $B: \Omega \rightarrow \mathbb{R}$. The interaction is given by the minimal coupling hypothesis, which requires the introduction of a real vector field $A=\left(A_{1}, A_{2}\right): \Omega \rightarrow \mathbb{R}^{2}$ such that $B=\partial_{1} A_{2}-\partial_{2} A_{1}$. Then the free energy of the system is equal to (1.2). In order that the free energy functional be well defined, we must impose some conditions on $\Phi$ and $A$.

Let us denote $H^{s, p}$ the usual Sobolev spaces of complex functions on $\Omega$ and $H^{s, 2} \equiv H^{s}$. Then we define $\underline{H}^{s, p}$ as the space of $\mathbb{C}^{2}$-valued functions on $\Omega$ with components in $H^{s, p}\left(\underline{H}^{s} \equiv \underline{H}^{s, 2}\right)$. $H_{\text {real }}^{s, p}$ (respectively $\left.\underline{H}_{\text {real }}^{s, p}\right)$ will denote the set of real (respectively $\mathbb{R}^{2}$ )-valued functions in $H^{s, p}$ (respectively $\left.\underline{H}^{s, p}\right)$. Let $\|\cdot\|_{p}$ be the norm in $L^{p} \equiv L^{p}(\Omega)$ and $\|\cdot\| \equiv\|\cdot\|_{2}$; we denote $|a|$ the modulus of the complex number $a$ or the euclidean norm of the complex vector $a \in \mathbb{C}^{2}$. 
The largest configuration space which we shall consider is the real Hilbert space:

$$
\mathscr{C}=\underline{H}_{\text {real }}^{1} \times H^{1} \text {. }
$$

Then the functional $\mathscr{A}_{\kappa}$ is well defined and continuous on $\mathscr{C}$; we may write:

$$
\mathscr{A}_{\kappa}(A, \Phi)=\frac{1}{2}\left\|D_{A} \Phi\right\|^{2}+\frac{1}{2}\left\|B_{A}\right\|^{2}+\frac{\kappa}{8}\left\||\Phi|^{2}-1\right\|^{2}
$$

and for $\kappa \geqq 0$ we have that $\mathscr{A}_{\kappa}$ is also positive. This follows from the Sobolev inclusion $H^{1} \subset \bigcap_{1 \leqq p<\infty} L^{p}$, that is true in two dimensions, and from the inequality $\|A \Phi\| \leqq\|A\|_{4} \cdot\|\Phi\|_{4}$. We remark that this configuration space is not the largest one making $\mathscr{A}_{\kappa}$ well defined, but is the largest one which has a simple mathematical description. In fact, because of the gauge invariance which we shall require below, the vector potential $A$ has no physical meaning but is introduced only in order to represent the magnetic field in the form $B=\partial_{1} A_{2}-\partial_{2} A_{1}$. A particular case of Theorem 4.2.2 from [4] shows that $A \mapsto B_{A}$ is a surjection of $\underline{H}^{s}$ onto $H^{s-1}$ for all $s \geqq 1$. Since the finiteness of $\mathscr{A}_{\kappa}$ requires $B \in L^{2}$, we can always find $A \in \underline{H}^{1}$ such that $B=B_{A}$. Then the finiteness of the last term in (2.2) implies that $\Phi \in L^{4}$. This shows that $D_{A} \Phi$ is a well defined distribution on $\Omega$ and (2.2) again implies that $D_{A} \Phi \in \underline{H}^{0}$. Since $A \in \underline{H}^{1}, A$ has components in $L^{p}$ for all $p<\infty$, hence $A \Phi \in \underline{H}^{0}$. Clearly then $D \Phi \in \underline{H}^{0}$, i.e. $\Phi \in H^{1}$ which shows that $\mathscr{C}$ is essentially the maximal configuration space which makes $\mathscr{A}_{\kappa}$ finite.

As we have mentioned in the introduction, we shall mainly consider perfect superconductors, for which the configuration space is given by:

$$
\mathscr{C}_{1}=\{(A, \Phi) \in \mathscr{C}|| \Phi \mid=1 \text { on } \Gamma\} .
$$

The definition makes sense because the restriction map $\varphi \mapsto \varphi \mid \Gamma$ has a continuous extension $H^{1}(\Omega) \rightarrow H^{1 / 2}(\Gamma)$. On $\mathscr{C}_{1}$ we consider the topology induced by that of $\mathscr{C}$. It is a non-trivial fact, following from the results of the appendix, that the configurations of $\mathscr{C}_{1}$ with $C^{\infty}(\bar{\Omega})$ components are dense in $\mathscr{C}_{1}$.

Proposition 2.1. The subset

$$
\mathscr{C}_{1, \infty}=\left\{(A, \Phi) \in \mathscr{C}_{1} \mid A \text { and } \Phi \text { are of class } C^{\infty}(\bar{\Omega})\right\}
$$

is dense in $\mathscr{C}_{1}$.

Proof. Since

$$
\mathscr{C}_{1}=\underline{H}_{\text {real }}^{1} \times\left\{\Phi \in H^{1}|| \Phi \mid=1 \text { on } \Gamma\right\}
$$

and $C^{\infty}(\bar{\Omega})$ is dense in $H^{1}(\Omega)$, it is enough to prove that each function $\Phi \in H^{1}$ with $|\Phi|=1$ on $\Gamma$ may be approached in $H^{1}$ by functions $\Phi_{n} \in C^{\infty}(\bar{\Omega})$ with $\left|\Phi_{n}\right|=1$ on $\Gamma$. Let $\varphi$ denote the trace of $\Phi$ on $\Gamma$. By the last part of Theorem A.3, there is a sequence $\left\{\varphi_{n}\right\}$ of functions belonging to $C^{\infty}(\Gamma)$ such that $\left|\varphi_{n}\right|=1$ and $\varphi_{n} \rightarrow \varphi$ in $H^{1 / 2}(\Gamma)$. It is known (see [15] Theorem 5, Chapter VI, Sect. 3) that there is a continuous linear application $R: H^{1 / 2}(\Gamma) \rightarrow H^{1}(\Omega)$ such that $R\left(C^{\infty}(\Gamma)\right) \subset C^{\infty}(\bar{\Omega})$ and $\left.R(f)\right|_{\Gamma}=f$ for all $f \in H^{1 / 2}(\Gamma)$. Let $\Psi_{n}=R\left(\varphi_{n}\right)$, so that $\Psi_{n} \rightarrow R(\varphi) \equiv \Psi$ in $H^{1}$ and $\Psi_{n} \in C^{\infty}(\bar{\Omega})$. Now $\Phi-\Psi \in H_{0}^{1}$, so we can find a sequence $\xi_{n} \in C_{0}^{\infty}(\Omega)$ such that $\xi_{n} \rightarrow \Phi-\Psi$ in $H^{1}$. Then $\Phi_{n}=\Psi_{n}+\xi_{n} \in C^{\infty}(\bar{\Omega}), \mid \Phi_{n} \|_{\Gamma}=1$ and $\Phi_{n} \rightarrow \Phi$ in $H^{1}$. 
We shall now refer to the so-called "gauge-invariance" of the system and the definition of the physical configuration space. We define the gauge-group as the additive group $\mathscr{G}=H_{\text {real }}^{2}$. It has a continuous action on $\mathscr{C}$ (leaving $\mathscr{C}_{1}$ invariant) given by:

$$
\mathscr{C} \ni(A, \Phi) \mapsto\left(A_{\Lambda}, \Phi_{\Lambda}\right) \stackrel{d}{=}\left(A+\nabla \Lambda, e^{i \Lambda} \Phi\right) \in \mathscr{C}
$$

for $\Lambda \in \mathscr{G}$. Then the physical configurations are in one to one correspondence with the orbits of this action, so that we can define the physical configuration space as $\hat{\mathscr{C}}=\mathscr{C} / \mathscr{G}$, or in the case of perfect superconductors $\hat{\mathscr{C}}_{1}=\mathscr{C}_{1} / \mathscr{G}$.

Let us observe that a function defined on the "mathematical" configuration space $\mathscr{C}\left(\right.$ or $\left.\mathscr{C}_{1}\right)$ is a physical quantity (i.e. depends only on the physical configuration) if and only if it is gauge-invariant (i.e. invariant under the action of $\mathscr{G}$ ). This is clearly true for $B_{A}$ and $|\Phi|$. Since $D_{A_{A}} \Phi_{\Lambda}=e^{i \Lambda} D_{A} \Phi$, this is also true for $\mathscr{A}_{\kappa}$ and for the current:

$$
J \equiv J(A, \Phi)=-\operatorname{Re}\left(\overline{D_{A} \Phi} \cdot \Phi\right)=\operatorname{Im}(\overline{\nabla \Phi} \cdot \Phi)+A|\Phi|^{2}
$$

The physical quantity defined here is in fact the current density of the pairs, which have a number density given by $|\Phi|^{2}$. This interpretation comes from a conservation law one may write for the configurations $(A, \Phi)$ which minimalize the free energy $\mathscr{A}_{\kappa}$, [14]. One should also remark that $B$ and $|\Phi|$ are not enough to uniquely characterize an orbit (i.e. a physical situation). This can be seen for example from the fact that $(A, \Phi) \mapsto(B,|\Phi|)$ is invariant under the action $(A, \Phi) \mapsto\left(A+\nabla \Lambda_{1}, e^{i \Lambda_{2}} \Phi\right)$ of the larger group $\mathscr{G} \times \mathscr{G}$, while $\left|D_{A} \Phi\right|$ is not.

We shall now make several remarks concerning the action of the gauge-group on $\mathscr{G}$. We recall a regularity result which will be repeatedly used in the following: if $\Lambda$ is a tempered distribution on $\Omega$ such that $\nabla \Lambda \in \underline{H}^{s}, s \in \mathbb{R} \backslash\left\{-\frac{1}{2},-\frac{3}{2}, \ldots\right\}$, then $\Lambda \in H^{s+1}$ (see [5]). In particular, if $A \in \underline{H}^{s}$, if $\Lambda$ is a tempered distribution on $\Omega$ and if $A+\nabla \Lambda \in C^{\infty}(\bar{\Omega})$, then $\Lambda \in H^{s+1}$. Also, this shows that in order that the action (2.4) of the gauge group leaves $\mathscr{C}$ invariant, one must define $\mathscr{G}$ as $H_{\text {real }}^{2}$. We would also like to remark that if $B_{A_{1}}=B_{A_{2}}$ then there exists at most one $\Lambda \in \mathscr{G}$ (up to an additive constant, which is irrelevant for us) such that $A_{2}=A_{1}+\nabla \Lambda$ ( $\Lambda$ exists if $\Omega$ is simply connected, but not in general).

The next two propositions contain some technical results which will be needed later on. We shall denote $v=\left(v_{1}, v_{2}\right): \Gamma \rightarrow S^{1} \subset \mathbb{R}^{2}$ (where $S^{1}$ is the unit circle) the exterior normal field on $\Gamma$ and $\tau=\left(\tau_{1}, \tau_{2}\right): \Gamma \rightarrow S^{1}$ the tangent field to $\Gamma$ defined by the condition that $\{v(x), \tau(x)\}$ is a positively oriented orthonormal basis in $\mathbb{R}^{2}$ for each $x \in \Gamma$. If $A \in \underline{H}_{\text {real }}^{1}$, then $\left.A\right|_{\Gamma}$ is a vector field on $\Gamma$ with components of class $H^{1 / 2}(\Gamma)$. Hence the scalar product $\left.v A \equiv v \cdot A\right|_{\Gamma} \in H^{1 / 2}(\Gamma)$ is well defined. We always denote by a dot the scalar product in $\mathbb{R}^{2}$ (extended by bilinearity to $\mathbb{C}^{2}$ ).

Proposition 2.2. Any configuration $\left(A^{\prime}, \Phi^{\prime}\right) \in \mathscr{C}$ is gauge-equivalent to a configuration $(A, \Phi) \in \mathscr{C}$ such that: $\operatorname{div} A=0$ and $v A=0$. Moreover, $A$ and $A^{\prime}$ have the same regularity properties; more precisely, if $A^{\prime} \in \underline{H}^{s, p}$ with $s \in \mathbb{N}$ and $1<p<\infty$ or any real $s \geqq 1$ and $p=2$, then $A \in \underline{H}^{s, p}$ also.

Proof. In fact, let us take an arbitrary configuration $\left(A^{\prime}, \Phi^{\prime}\right) \in \mathscr{C}$ and consider a gauge transformation $\Lambda \in \mathscr{G}$ and the new configuration $(A, \Phi)$ given by: $A=A^{\prime}+\nabla \Lambda$, 
$\Phi=\Phi^{\prime} e^{i \Lambda}$. Then, in order that $(A, \Phi)$ verify the described relations, we must have

$$
\left\{\begin{array}{l}
\operatorname{div} A=\operatorname{div} A^{\prime}+\Delta \Lambda=0 \\
v A=v A^{\prime}+v \nabla \Lambda=0,
\end{array}\right.
$$

where $v \nabla \Lambda \equiv \frac{\partial \Lambda}{\partial v}$, and thus $\Lambda$ must be the solution of a Neumann problem:

$$
\left\{\begin{array}{l}
\Delta \Lambda=-\operatorname{div} A^{\prime} \\
\frac{\partial \Lambda}{\partial v}=-v A^{\prime} .
\end{array}\right.
$$

Now this problem has a unique solution up to an additive constant, because: $\operatorname{div} A^{\prime} \in L^{2}(\Omega), v A^{\prime} \in H^{1 / 2}(\Gamma)$ and the compatibility condition (we denote $d \sigma$ the length element on $\Gamma)$ :

$$
\int_{\Omega} \operatorname{div} A^{\prime} d x=\int_{\Gamma} v A^{\prime} d \sigma
$$

is evidently satisfied. Then the proposition follows from [9].

Remark. The gauge transformation $\Lambda$ which relates $(A, \Phi)$ to $\left(A^{\prime}, \Phi^{\prime}\right)$ in the preceding proposition is unique up to an additive constant.

A configuration $(A, \Phi) \in \mathscr{C}$ is said to be locally gauge-equivalent to $C^{\infty}$ configurations if for each $x \in \bar{\Omega}$ there is an open neighbourhood $U$ of $x$ in $\mathbb{R}^{2}$ and a function $\Lambda=\Lambda_{U} \in H_{\text {real }}^{2}(U)$ such that $A+\nabla \Lambda$ and $e^{i \Lambda} \Phi$ are class $C^{\infty}$ on $\bar{\Omega} \cap U$.

Proposition 2.3. If a configuration $(A, \Phi) \in \mathscr{C}$ is locally gauge-equivalent to $C^{\infty}$ configurations, then it is gauge-equivalent to a configuration of class $C^{\infty}$ on $\bar{\Omega}$ (i.e. there is $\Lambda \in \mathscr{G}$ such that $A+\nabla \Lambda$ and $e^{i \Lambda} \Phi$ are $C^{\infty}$ functions on $\bar{\Omega}$ ).

Proof. All gauge-invariant quantities associated to $(A, \Phi)$ are clearly $C^{\infty}$ on $\bar{\Omega}$. In particular $B=\partial_{1} A_{2}-\partial_{2} A_{1} \in C^{\infty}(\bar{\Omega})$. If we denote $d A$ the exterior derivative of the vector field $A$ (we identify vector fields with 1 -forms and 2 -forms with functions) then we can also write $B=d A$. Known results (see for example [4]) imply that the equation $d X=B$ will have solutions $X$ of class $C^{\infty}(\bar{\Omega})$. Two solutions $X^{\prime}, X^{\prime \prime}$ of class $\underline{H}^{1}$ will have the property $d\left(X^{\prime}-X^{\prime \prime}\right)=0$. Using once again results from [4] for example, it follows that there is a function $f \in H^{2}$ and a vector field $Y$ of class $C^{\infty}(\bar{\Omega})$ such that $X^{\prime}-X^{\prime \prime}=d f+Y$ ( $Y$ is a closed but not exact form). In conclusion there is $\Lambda \in H_{\text {real }}^{2}$ such that $A+d \Lambda$ is of class $C^{\infty}(\bar{\Omega})$. Let us now consider an open neighbourhood $U$ and a function $\Lambda_{U}$ as in the above definition. Then $(A+d \Lambda)-\left(A+d \Lambda_{U}\right)$ is of class $C^{\infty}$ on $U \cap \bar{\Omega}$, hence $d\left(\Lambda-\Lambda_{U}\right)$ has the same property. Clearly this implies that $\Lambda-\Lambda_{U}$ is $C^{\infty}$ on $U \cap \bar{\Omega}$. This being true by hypothesis for $\exp \left(i \Lambda_{U}\right) \cdot \Phi$, it will also be true for $\exp (i \Lambda) \Phi$. Since the sets $U$ cover $\bar{\Omega}$, we finally get $\exp (i \Lambda) \Phi \in C^{\infty}(\bar{\Omega})$.

In the following we shall define a certain gauge-invariant quantity which may be given some interesting physical interpretation in connection with the so-called London effect of magnetic flux quantization. In [8] Jaffe and Taubes study the magnetic flux inside the superconductor (of type II) and prove that its values are 
only integer multiplies of a certain quantity. In our case the problem is complicated by the presence of the boundaries and the boundary conditions which in physical terms are determinated by the field configuration outside the superconducting material. Making use of the current defined in (2.5) we may define the following gauge-invariant, and thus physically relevant, quantity:

$$
\mathscr{F}(A, \Phi)=\frac{1}{2 \pi} \int_{\Omega} B_{A} d x-\frac{1}{2 \pi} \int_{\Gamma} \tau \cdot J(A, \Phi) d \sigma .
$$

As we already said, $\tau \cdot J$ is the scalar product in $\mathbb{R}^{2}$ of the vectors $\tau$ and $J$, and $d \sigma$ is the measure on $\Gamma$ induced by the euclidean structure of $\mathbb{R}^{2}$. We interpret $\mathscr{F}(A, \Phi)$ as a magnetic flux inside $\Omega$, this being in agreement with the second London equation (see formula (1.11) in [12]) which allows us to give a physical meaning to the last term in (2.8). On the other hand it is not evident that this term is mathematically well defined. In fact, $A$ and $\Phi$ being of class $H^{1}$, their restrictions to $\Gamma$ make sense and are functions of class $H^{1 / 2}(\Gamma)$ (see [10]). However, the restrictions of the derivatives $\partial_{j} \Phi$ to $\Gamma$ does not have any meaning for a general configuration, hence some problems arise in connection with the term $\operatorname{Im}(\bar{\nabla} \Phi \cdot \Phi)$ from (2.5). In order to define (2.8) for a general configuration $(A, \Phi) \in \mathscr{C}$, remark first that $\tau \cdot \nabla \Phi=\frac{d \Phi}{d \sigma}$ for $C^{1}$-functions $\Phi$ on $\Gamma$, where we have denoted $\frac{d}{d \sigma}$ the tangential derivative operator on $\Gamma$. Denote $\langle\cdot,\rangle_{\Gamma}: H^{1 / 2}(\Gamma) \times H^{-1 / 2}(\Gamma) \rightarrow \mathbb{C}$ the antiduality map (antilinear in the first variable) obtained from the scalar product in $L^{2}(\Gamma)$ by considering the continuous, linear embeddings $H^{1 / 2}(\Gamma) \subset L^{2}(\Gamma) \subset$ $H^{-1 / 2}(\Gamma)$ (i.e. $\langle f, g\rangle_{\Gamma}=\int_{\Gamma} \bar{f} g d \sigma$ if $\left.f, g \in L^{2}(\Gamma)\right)$. Then we define $\mathscr{F}: \mathscr{C} \rightarrow \mathbb{R}$ by:

$$
\mathscr{F}(A, \Phi)=\frac{1}{2 \pi} \int_{\Omega} B_{A} d x+\frac{1}{2 \pi} \operatorname{Im}\left\langle\Phi, \frac{d \Phi}{d \sigma}\right\rangle_{\Gamma}-\frac{1}{2 \pi} \int_{\Gamma} \tau \cdot A|\Phi|^{2} d \sigma .
$$

Since the operation of restriction $H^{1}(\Omega) \rightarrow H^{1 / 2}(\Gamma)$ and the derivation $\frac{d}{d \sigma}: H^{1 / 2}(\Gamma) \rightarrow H^{-1 / 2}(\Gamma)$ are continuous, and $H^{1 / 2}(\Gamma) \subset L^{p}(\Gamma)$ for all $p<\infty$, we see that $\mathscr{F}$ is a continuous functional on the Banach space $\mathscr{C}$. From the usual integration by parts formula (valid for any $A \in \underline{H}^{1}$ ) we get:

$$
\int_{\Omega} B_{A} d x=\int_{\Gamma}\left(v_{1} A_{2}-v_{2} A_{1}\right) d \sigma=\int_{\Gamma}(* v) \cdot A d \sigma=\int_{\Gamma} \tau \cdot A d \sigma,
$$

and thus we can put (2.9) in the following form:

$$
\mathscr{F}(A, \Phi)=\frac{1}{2 \pi} \operatorname{Im}\left\langle\Phi, \frac{d \Phi}{d \sigma}\right\rangle_{\Gamma}+\frac{1}{2 \pi} \int_{\Gamma} \tau \cdot A\left(1-|\Phi|^{2}\right) d \sigma .
$$

If we restrict ourselves to configurations in $\mathscr{C}_{1}$, the last term vanishes and we see that $\mathscr{F}(A, \Phi)$ equals the topological degree of the function $\left.\Phi\right|_{\Gamma}: \Gamma \rightarrow S^{1}$ of class $H^{1 / 2}$, which is an integer. This is a consequence of Theorem A.3 of the Appendix if we take into account that $\Gamma$ is a finite union of curves diffeomorphic to $S^{1}$. It is also an immediate consequence of Proposition 2.1 because $\mathscr{F}$ is continuous on $\mathscr{C}$ and $\mathscr{F}(A, \Phi)$ is trivially an integer if $(A, \Phi) \in \mathscr{C}_{1, \infty}$. Since $\mathscr{F}: \mathscr{C}_{1} \rightarrow \mathbb{Z}$ and is 
continuous, we see that the configuration space $C_{1}$ is disconnected. We summarize these results in:

Proposition 2.4. The functional $\mathscr{F}$ defined by (2.9) (formally by (2.8)) on $\mathscr{C}$ is continuous. Its restriction to $\mathscr{C}_{1}$ is given by

$$
\mathscr{F}(A, \Phi)=\frac{1}{2 \pi} \operatorname{Im}\left\langle\Phi, \frac{d \Phi}{d \sigma}\right\rangle_{\Gamma} \equiv \frac{1}{2 \pi i}\left\langle\Phi, \frac{d \Phi}{d \sigma}\right\rangle_{\Gamma},
$$

which is equal to the topological degree of the map $\left.\Phi\right|_{\Gamma}: \Gamma \rightarrow S^{1}$. In particular, $\mathscr{F} \mid \mathscr{C}_{1}$ has only integral values, the sets $\mathscr{C}_{1}^{N}=\left\{(A, \Phi) \in \mathscr{C}_{1} \mid \mathscr{F}(A, \Phi)=N\right\}(N \in \mathbb{Z})$ are open and closed in $\mathscr{C}_{1}, \mathscr{C}_{1}^{N} \cap \mathscr{C}_{1}^{M}=\phi$ for $N \neq M$ and $\mathscr{C}_{1}=\bigcup_{N \in \mathbb{Z}} \mathscr{C}_{1}^{N}$.

The subsets $\mathscr{C}_{1}^{N}$ are not connected if $\Omega$ is not simply connected. We shall now find the connected components of $\mathscr{C}_{1}$. Let $\Gamma_{0}, \Gamma_{1}, \ldots, \Gamma_{m}(m \geqq 0)$ be the connected components of $\Gamma, \Gamma_{0}$ being the exterior boundary of $\Omega$. Each $\Gamma_{j}$ is diffeomorphic to $S^{1}$, hence if $f: \Gamma_{j} \rightarrow S^{1}$ is of class $H^{1 / 2}$ one can define its topological degree (or winding number) which will be an integer denoted $G(f)$ (see Theorem A.3 of the Appendix). If $f: \Gamma \rightarrow S^{1}$ is of class $H^{1 / 2}$, put $G(f)=\sum_{j=0}^{m} G\left(f .\left.\right|_{\Gamma_{j}}\right)$ (remark that
the orientation of $\Gamma_{j}$ is determined by $\Omega$ ). Clearly

$$
\mathscr{F}(A, \Phi)=G\left(\left.\Phi\right|_{\Gamma}\right)=\sum_{j=0}^{m} \frac{1}{2 \pi i}\left\langle\Phi, \frac{d \Phi}{d \sigma}\right\rangle_{\Gamma_{j}}=\sum_{j=0}^{m} G\left(\left.\Phi\right|_{\Gamma_{j}}\right) .
$$

The functions $\mathscr{C}_{1} \ni(A, \Phi) \mapsto G\left(\left.\Phi\right|_{\Gamma_{j}}\right) \in \mathbb{Z}$ are clearly continuous. If $N_{0}, N_{1}, \ldots, N_{m}$ are integers, let us denote $\mathscr{C}_{1}^{N_{0}}, \ldots, N_{m}$ the set of elements $(A, \Phi) \in \mathscr{C}_{1}$ such that $G\left(\left.\Phi\right|_{\Gamma_{j}}\right)=N_{j}$ for all $j$. Then $\mathscr{C}_{1}^{N_{0}, \ldots, N_{m}}$ are open and closed in $\mathscr{C}_{1}$, and $\mathscr{C}_{1}$ is the disjoint union of all sets $\mathscr{C}_{1}^{N_{0}}, \ldots, N_{m}$.

Proposition 2.5. $\mathscr{C}_{1}^{N_{0}, \ldots, N_{m}}$ is connected.

Proof. By Proposition 2.1 and the continuity of $(A, \Phi) \mapsto G\left(\left.\Phi\right|_{\Gamma_{j}}\right)$, the subset of $\mathscr{C}_{1}^{N_{0}, \ldots, N_{m}}$ of configurations with $C^{\infty}(\bar{\Omega})$ components is a dense subset of $\mathscr{C}_{1}^{N_{0}, \ldots, N_{m}}$. Hence, by an argument similar to that in the proof of Proposition 2.1, it is enough to prove that the set:

$$
\left\{\Phi \in C^{\infty}(\bar{\Omega})|| \Phi \mid=1 \text { on } \Gamma \text { and } G\left(\left.\Phi\right|_{\Gamma_{j}}\right)=N_{j}, j=0, \ldots, m\right\}
$$

is a connected subset of $C^{\infty}(\bar{\Omega})$. Let $\Phi_{0}, \Phi_{1}$ be two functions in this subset and $d>0$ small enough. Denote $U=\{x \in \Omega \mid$ dist. $(x, \Gamma)<d\}$ and remark that we can choose $d$ so that $\left|\Phi_{i}\right|>1 / 2$ on $U$ for $i=0,1$. Clearly (for $d$ small) $U$ is the disjoint union of open sets diffeomorphic to $\{z \in \mathbb{C}|1<| z \mid<2\}$. Since $\psi=\Phi_{1} \Phi_{0}^{-1} \in C^{\infty}(\bar{U})$, $|\psi| \geqq$ const. $>0,|\psi|=1$ on $\Gamma$ and $G\left(\psi \mid \Gamma_{j}\right)=0$ for all $j$, it follows that there is a function $g \in C^{\infty}(\bar{U})$ which is real on $\Gamma$ such that $\psi=\exp i g$. Now let $a, b \in C^{\infty}(\bar{\Omega})$ with supp $a \subset \bar{U}, a=1$ in a neighbourhood of $\Gamma$ and $a+b=1$ on $\bar{\Omega}$. Since $\Phi_{1}=a \Phi_{1}+b \Phi_{1}=a e^{i g} \Phi_{0}+b \Phi_{1}$, the application $t \mapsto \Phi_{t}=a e^{i t g} \Phi_{0}+b\left(t \Phi_{1}+(1-t) \Phi_{0}\right)$ is a continuous path in the set (2.13) going from $\Phi_{0}$ to $\Phi_{1}$.

Remark that the gauge group $\mathscr{G}$ on $\mathscr{C}_{1}$ leaves invariant $\mathscr{C}_{1}^{N_{0}, \ldots, N_{m}}$, hence the physical configuration space $\mathscr{C}_{1}$ has a similar connectedness structure. 


\section{Smoothness of the Stationary Configurations}

In this chapter we prove analyticity (modulo a gauge transformation) of the stationary points of the functional $\mathscr{A}_{\kappa}$ defined on $\mathscr{C}$ or on a subset of $\mathscr{C}$ defined by boundary conditions on $\Gamma$ only (for example on $\mathscr{C}_{1}$ ). Then we show that the stationary points of $\mathscr{A}_{\kappa} \mid \mathscr{C}_{1}$ satisfy some interesting relations which can be interpreted in physical terms as Meissner effect (we follow Taubes, see [18]).

We begin by calculating the first and second derivatives of the continuous functional $\mathscr{A}_{\kappa}: \mathscr{C} \rightarrow \mathbb{R}\left(\mathscr{C}\right.$ is a real Hilbert space) by expanding $\mathscr{A}_{\kappa}(A+a, \Phi+\varphi)$ around $(A, \Phi) \in \mathscr{C}$ for $(a, \varphi) \in \mathscr{C}$. The first derivative, at the point $(A, \Phi)$, is the linear $\operatorname{map} \mathscr{A}_{\kappa}^{\prime}(A, \Phi): \mathscr{C} \rightarrow \mathbb{R}$ given by

$$
\mathscr{A}_{\kappa}^{\prime}(A, \Phi)(a, \varphi)=\operatorname{Re}\left\langle D_{A} \Phi, D_{A} \varphi-a \Phi\right\rangle+\left\langle B_{A}, B_{a}\right\rangle-\kappa \operatorname{Re}\left\langle\frac{1-|\Phi|^{2}}{2} \Phi, \varphi\right\rangle,
$$

where $\langle\cdot, \cdot\rangle$ is the scalar product (anti-linear in the first variable) in $L^{2}(\Omega)$ (or in the corresponding space of $\mathbb{C}^{2}$-valued functions). The second derivative is the symmetric bilinear map $\mathscr{A}_{\kappa}^{\prime \prime}(A, \Phi): \mathscr{C} \times \mathscr{C} \rightarrow \mathbb{R}$ defined by:

$$
\begin{aligned}
\mathscr{A}_{\kappa}^{\prime \prime}(A, \Phi)((a, \varphi),(a, \varphi))= & \left\|D_{A} \varphi\right\|^{2}-2 \operatorname{Re}\left\langle D_{A} \varphi, a \Phi\right\rangle+\|a \Phi\|^{2} \\
& -2 \operatorname{Re}\left\langle D_{A} \Phi, a \varphi\right\rangle+\frac{1}{2}\left\|B_{a}\right\|^{2}+\frac{\kappa}{2}\|\operatorname{Re} \varphi \bar{\Phi}\|^{2} \\
& -\frac{\kappa}{2}\left\langle\frac{1-|\Phi|^{2}}{2},|\varphi|^{2}\right\rangle .
\end{aligned}
$$

By stationary configuration we shall mean a configuration $(A, \Phi) \in \mathscr{C}$ such that $\mathscr{A}_{\kappa}^{\prime}(A, \Phi)(a, \varphi)=0$ if $(a, \varphi)$ has components in $C_{0}^{\infty}(\Omega)$. This is weaker than asking that the derivative $\mathscr{A}_{\kappa}^{\prime}(A, \Phi)$ be equal to zero. Moreover, if we restrict $\mathscr{A}_{\kappa}$ to a submanifold $\mathscr{D}$ of $\mathscr{C}$ with the property $(A+a, \Phi+\varphi) \in \mathscr{D}$ if $(A, \Phi) \in \mathscr{D}$ and $(a, \varphi)$ has components in $C_{0}^{\infty}(\Omega)$ (for example $\mathscr{D}=\mathscr{C}_{1}$ ), then the points where the derivative of the restriction is zero are stationary configurations (because the tangent space to $\mathscr{D}$ at some point $(A, \Phi) \in \mathscr{D}$ will contain the configurations with components of class $C_{0}^{\infty}(\Omega)$ ).

From (3.1) and a similar relation but with $\varphi$ replaced by $i \varphi$, we see that $(A, \Phi)$ is a stationary configuration if and only if:

$$
\left\langle D_{A} \Phi, D_{A} \varphi\right\rangle-\kappa\left\langle\frac{1-|\Phi|^{2}}{2} \Phi, \varphi\right\rangle+\left\langle B_{A}, B_{a}\right\rangle-\left\langle\operatorname{Re}\left(\Phi \overline{D_{A} \Phi}\right), a\right\rangle=0
$$

for all $(a, \varphi)$ with $C_{0}^{\infty}(\Omega)$ components. This condition is equivalent to the following system of second order differential equations for $(A, \Phi)$ :

$$
\left\{\begin{array}{l}
D_{A}^{2} \Phi=\kappa \frac{1-|\Phi|^{2}}{2} \Phi \\
*\left(\nabla B_{A}\right)=-\operatorname{Re}\left(\Phi \overline{D_{A} \Phi}\right) \equiv J(A, \Phi) .
\end{array}\right.
$$

All the terms of these equations are well defined because $(A, \Phi)$ has components 
in $H^{1} \subset \bigcap_{p<\infty} L^{p}$. In particular we obtain that $J(A, \Phi)$, and hence $\nabla B_{A}$, has components of class $L^{q}(\Omega)$ for all $q<2$. This implies that $B_{A} \in H^{1, q}$ for all $q<2$.

Theorem 3.1. Each stationary configuration $(A, \Phi) \in \mathscr{C}$ has the property $\Phi \in H_{\text {loc }}^{2}(\Omega)$ and is gauge-equivalent to a configuration with real-analytic components on $\Omega$. More precisely, if $(A, \Phi) \in \mathscr{C}$ is stationary and $\operatorname{div} A=0$ (which can always be achieved by a gauge transformation, see Proposition 2.2), then $A$ and $\Phi$ are real-analytic functions on $\Omega$.

Proof. In the case $\operatorname{div} A=0$ the system (3.4) becomes

$$
\left\{\begin{array}{l}
D^{2} \Phi=2 A \cdot D \Phi-A^{2} \Phi+\kappa \frac{1-|\Phi|^{2}}{2} \Phi \\
D^{2} A=\operatorname{Re}\left(\Phi \overline{D_{A} \Phi}\right)-A|\Phi|^{2}
\end{array}\right.
$$

which is an elliptic system and $(A, \Phi)$ has components in $H^{1}$. We can obtain the analyticity of the solution by directly applying a rather difficult theorem of Morrey [11]. We shall give a simple proof of the fact that the solutions are of class $C^{\infty}(\Omega)$ based on the well-known lemma: if $T$ is a distribution on $\Omega$ such that $\Delta T \in L_{\text {loc }}^{p}$ for some $p \in(1, \infty)$, then $T \in H_{\text {loc }}^{2, p}$. This immediately implies that $(A, \Phi)$ has components in $H_{\text {loc }}^{2, q}$ for all $q<2$. Since $H_{\text {loc }}^{2, q} \subset L_{\text {loc }}^{\infty}$ for $2-q$ small enough, we see that $(A, \Phi)$ has components in $L_{\text {loc }}^{\infty}$. Returning to Eqs. (3.5) we observe that $D^{2} \Phi$ and $D^{2} A$ are of class $L_{\text {loc }}^{2}$, hence $(A, \Phi)$ has components in $H_{\text {loc }}^{2}$. Now by differentiating Eqs. (3.5) and by an iteration procedure we get that the components of $(A, \Phi)$ are in $H_{\text {loc }}^{k}$ for all $k \in \mathbb{N}$, hence are of class $C^{\infty}(\Omega)$.

We pass now to the mathematical formulation of the Meissner effect (see Jaffe and Taubes [8 and 12-14]). Now the boundary conditions play an important role. The following argument justifies our choice. We consider only the restriction of the functional $\mathscr{A}_{\kappa}$ to the set $\mathscr{C}_{1}$. The tangent space to $\mathscr{C}_{1}$ at a point $(A, \Phi) \in \mathscr{C}_{1}$ is the linear subspace of $\mathscr{C}$ consisting of configurations $(a, \varphi)$ such that $\left.\operatorname{Re} \varphi \bar{\Phi}\right|_{\Gamma}=0$ (no condition on $a$ ). Taking $\varphi=0$ in (3.3) we see that in order that the derivative of $\mathscr{A}_{\kappa} \mid \mathscr{C}_{1}$ be zero at $(A, \Phi)$ one must have:

$$
\left\langle B_{A}, B_{a}\right\rangle+\langle J(A, \Phi), a\rangle=0
$$

for all $a \in H_{\text {real }}^{1}$. We already know that for a stationary configuration one must have $B_{A} \in H^{1, q}$ for all $q<2$, hence the restriction $B_{A} \mid \Gamma$ makes sense as an element of $L^{2}(\Gamma)$ and if $a$ is of class $C^{1}(\Omega)$ :

$$
\left\langle B_{A}, B_{a}\right\rangle=\left\langle-* \nabla B_{A}, a\right\rangle+\int_{\Gamma} B_{A} \tau \cdot a d \sigma .
$$

From (3.6), (3.7), the second equation (3.4) and the arbitrariness of $a$ we get $B_{A} \mid \Gamma=0$. This explains the conditions we put in the next definition:

Definition. $(A, \Phi)$ is a $\mathscr{C}_{1}$-regular stationary configuration if $\Phi \in H^{1} \cap L^{\infty}, \mid \Phi \|_{\Gamma}=1$, $B_{A} \in H^{1}, B_{A} \mid \Gamma=0$ and is a stationary configuration of $\mathscr{A}_{\kappa}$ (remark that these conditions are gauge-invariant). 
Theorem 3.2. If $(A, \Phi)$ is a $\mathscr{C}_{1}$-regular stationary configuration, then:

$1^{\circ}$ Either $|\Phi|=1$ on $\Omega$, or $|\Phi(x)|<1$ for all $x \in \Omega$. $2^{\circ}$ If, moreover, $\kappa \leqq 1$, then either $\left|B_{A}\right|=\frac{1-|\Phi|^{2}}{2}$ on $\Omega$ or $\left|B_{A}(x)\right|<\frac{1-|\Phi(x)|^{2}}{2}$ for
all $x \in \Omega$.

Proof. We essentially follow Sect. III.8 of [8]. Let $w=\frac{1}{2}\left(1-|\Phi|^{2}\right) \in H_{0}^{1}(\Omega)$. The first equation (3.4) gives

$$
\left(D^{2}+\kappa|\Phi|^{2}\right) w=\left|D_{A} \Phi\right|^{2} \geqq 0 .
$$

The maximum principle (Theorem 8.1 in [7]) implies now the first assertion of the theorem. Then, the second equation (3.4) gives:

$$
\left(D^{2}+|\Phi|^{2}\right) B_{A}=i\left(\overline{D_{A} \Phi}\right) \cdot\left(* D_{A} \Phi\right),
$$

where $\left(a_{1}, b_{1}\right) \cdot\left(a_{2}, b_{2}\right)=a_{1} a_{2}+b_{1} b_{2}$ for two vectors in $\mathbb{C}^{2}$. If $\kappa \leqq 1$, we get from (3.8) and (3.9):

$$
\left(D^{2}+|\Phi|^{2}\right)\left(w \pm B_{A}\right)=(1-\kappa)|\Phi|^{2} w+\left|D_{A} \Phi\right|^{2} \pm i\left(\overline{D_{A} \Phi}\right) \cdot\left(* D_{A} \Phi\right) \geqq 0 .
$$

Since $w, B \in H_{0}^{1}(\Omega)$, a new application of the maximum principle gives the second assertion of the theorem.

The fact that the magnetic field $B_{A}$ vanishes in the super-conducting phase $(|\Phi|=1)$ is called Meissner effect in the physics literature.

\section{Vortex-Antivortex Type Configurations}

In this chapter we shall apply the techniques of Bogomol'nyi [2] and Jaffe and Taubes [8] for a detailed study of some of the stationary points of $\mathscr{A}_{\kappa}$ in the critical case $\kappa=1$ (see the introduction). The general idea is that of Taubes, the main differences being due to the following situation. In the case studied by Taubes $\left(\Omega=\mathbb{R}^{2}\right)$, the stationary configurations were globally smooth, but there were difficulties due to the unboundedness of $\mathbb{R}^{2}$. In our case $\Omega$ is bounded, but we have not been able to show regularity up to the boundary (i.e. on $\bar{\Omega}$ ) of the stationary configurations of $\mathscr{A}_{\kappa}$. So, one of the main points of this chapter will be to study the boundary behaviour of the absolute minima of $\mathscr{A}_{1} \mid \mathscr{C}_{1}^{N}$ for all $N \in \mathbb{Z}$, see Proposition 4.2. Then we shall give a complete classification of the minima (Theorem 4.4).

It was an important remark of Bogomol'nyi that in the critical case $\kappa=1$ one may replace the second-order non-linear system (3.4) by a first-order system which can then be studied much more easily using the methods of Taubes. The first step is to put the functional $\mathscr{A}_{\kappa}$ into a form which involves explicitly the total flux $\mathscr{F}$ and gives better lower bounds for $\mathscr{A}_{\kappa}$ on the open sets $\mathscr{C}_{1}^{N}, N \in \mathbb{Z}$. For this, recall that $*: \mathbb{C}^{2} \rightarrow \mathbb{C}^{2}$ is given by $*\left(\begin{array}{l}a_{1} \\ a_{2}\end{array}\right)=\left(\begin{array}{c}-a_{2} \\ a_{1}\end{array}\right)$ (from now on we think of the elements of $\mathbb{C}^{2}$ as column vectors and we provide $\mathbb{C}^{2}$ with the usual scalar product, antilinear in the first variable: $\left.\langle a \mid b\rangle=\bar{a}_{1} b_{1}+\bar{a}_{2} b_{2}\right)$. Thus $*$ is the unitary operator in $\mathbb{C}^{2}$ defined by the matrix $*=\left(\begin{array}{cc}0 & -1 \\ 1 & 0\end{array}\right)$. 
If $p_{ \pm}=\frac{1 \mp i *}{2}=\frac{1}{2}\left(\begin{array}{cc}1 & \pm i \\ \mp i & 1\end{array}\right)$, then $p_{ \pm}$are orthogonal projections in $\mathbb{C}^{2}, p_{+}$is orthogonal to $p_{-}$and $*=i p_{+}-i p_{-}$. This implies:

$$
\begin{aligned}
-i\left\langle D_{A} \Phi, * D_{A} \Phi\right\rangle & =\left\|p_{+} D_{A} \Phi\right\|^{2}-\left\|p_{-} D_{A} \Phi\right\|^{2}, \\
\left\|D_{A} \Phi\right\|^{2} & =\left\|p_{+} D_{A} \Phi\right\|^{2}+\left\|p_{-} D_{A} \Phi\right\|^{2} .
\end{aligned}
$$

Assume for the moment $\Phi \in H^{2}$ and integrate by parts the left-hand side of (4.1). Since $D_{A} \cdot * D_{A}=-\left(D_{A}\right)_{1}\left(D_{A}\right)_{2}+\left(D_{A}\right)_{2}\left(D_{A}\right)_{1}=-i B_{A}$ and $* v(x)=\tau(x)$ for each $x \in \Gamma$, we get

$$
\begin{aligned}
-i\left\langle D_{A} \Phi, * D_{A} \Phi\right\rangle & =-i\left\langle\Phi, D_{A} \cdot * D_{A} \Phi\right\rangle+\int_{\Gamma} \bar{\Phi}\left[v \cdot * D_{A} \Phi\right] d \sigma \\
& =-\left\langle\Phi, B_{A} \Phi\right\rangle-\int_{\Gamma} \bar{\Phi}\left[* v \cdot D_{A} \Phi\right] d \sigma \\
& =-\left\langle|\Phi|^{2}, B_{A}\right\rangle-\int_{\Gamma} \bar{\Phi}_{\tau} \cdot D_{A} \Phi d \sigma .
\end{aligned}
$$

Since this is a real quantity (see (4.1)), taking the real part and using (2.5), (2.8), we get

$$
-i\left\langle D_{A} \Phi, * D_{A} \Phi\right\rangle=\left\langle 1-|\Phi|^{2}, B_{A}\right\rangle-2 \pi \mathscr{F}(A, \Phi) .
$$

We know that $\mathscr{F}: \mathscr{C}_{1} \rightarrow \mathbb{R}$ is continuous and that configurations with $C^{\infty}(\bar{\Omega})$ components are dense in $\mathscr{C}$. Hence (4.3) remains true for all $(A, \Phi) \in \mathscr{C}_{1}$ (not only for those with $\Phi \in H^{2}$ ). Then (4.1), (4.2) and (4.3) imply:

$$
2\left\|p_{ \pm} D_{A} \Phi\right\|^{2}=\left\|D_{A} \Phi\right\|^{2} \pm\left\langle 1-|\Phi|^{2}, B_{A}\right\rangle \mp 2 \pi \mathscr{F}(A, \Phi) .
$$

This allows us to put (2.2) in the following form:

$$
\mathscr{A}_{\kappa}(A, \Phi)=1 / 4\left\|D_{A} \Phi \mp i * D_{A} \Phi\right\|^{2}+1 / 2\left\|B_{A} \mp w\right\|^{2}+(\kappa-1) / 2\|w\|^{2} \pm \pi \mathscr{F}(A, \Phi)
$$

for all $(A, \Phi) \in \mathscr{C}_{1}$. The notation $w=1 / 2\left(1-|\Phi|^{2}\right)$ will be systematically used from now on.

Let us restrict ourselves to the critical case $\kappa=1$. Since $\mathscr{C}_{1}$ is the disjoint union of the open subsets $\mathscr{C}_{1}^{N}, N \in \mathbb{Z}$ (see Proposition 2.3), the stationary configurations of $\mathscr{A}_{1} \mid \mathscr{C}_{1}$ can be found by studying the restrictions $\mathscr{A}_{1} \mid \mathscr{C}_{1}^{N}$. Let us fix an integer $n \geqq 0$ and consider for $\mathscr{A}_{1} \mid \mathscr{C}_{1}^{ \pm n}$ the form (4.5). Since $\mathscr{F} \mid \mathscr{C}_{1}^{ \pm n}= \pm n$, we get:

$$
\mathscr{A}_{1}(A, \Phi)=1 / 4\left\|D_{A} \Phi \mp i * D_{A} \Phi\right\|^{2}+1 / 2\left\|B_{A} \mp w\right\|^{2}+n \pi, \text { for all }(A, \Phi) \in \mathscr{C}_{1}^{ \pm n} .
$$

From the above representation one sees that $\mathscr{A}_{1}$ on $\mathscr{C}_{1}^{ \pm n}$ is bounded below by $\pi n$ and this minimum is effectively reached for those configurations $(A, \Phi) \in \mathscr{C}_{1}^{ \pm n}$ which satisfy the following system of first-order non-linear equations:

$$
\left\{\begin{array}{l}
\left(D_{A} \mp i * D_{A}\right) \Phi=0 \\
B_{A}= \pm w \equiv \pm 1 / 2\left(1-|\Phi|^{2}\right)
\end{array} .\right.
$$

Remark. $\mathscr{C}_{1}^{ \pm n}$ being open subsets of $\mathscr{C}_{1}$, any minimum of $\mathscr{A}_{1}$ in $\mathscr{C}_{1}^{ \pm n}$ is also a minimum of $\mathscr{A}_{1}$ in $\mathscr{C}_{1}$, and thus any solution of (4.7) is a stationary configuration 
of $\mathscr{A}_{1}$, i.e. verifies (3.4) with $\kappa=1$ (of course this can also be proved by direct calculation). The following can be shown (we do not give details, because this is outside the main subject of the paper): a $\mathscr{C}_{1}$-regular stationary configuration $(A, \Phi)$ of $\mathscr{A}_{1}$ in $\mathscr{C}_{1}^{ \pm n}$ with $\Phi \in H^{2}$ satisfies (4.7) (i.e. is an absolute minimum of $\mathscr{A}_{1} \mid \mathscr{C}_{1}^{ \pm n}$ ) if and only if

$$
\int_{\Gamma} v \cdot x\left[(\partial|\Phi| / \partial v)^{2}-1 / 2\left|D_{A} \Phi\right|^{2}\right] d \sigma=0
$$

The configurations $(A, \Phi) \in \mathscr{C}_{1}^{ \pm n}$ on which $\mathscr{A}_{1} \mid \mathscr{C}_{1}^{ \pm n}$ assumes its absolute minimum $\pi n$ are the object of our study from now on. We shall confine ourselves to the case $\mathscr{A}_{1} \mid \mathscr{C}_{1}^{n}$, the other case being obviously similar.

So, let us fix an integer $n \geqq 0$. A configuration $(A, \Phi) \in \mathscr{C}_{1}^{n}$ realizes the absolute minimum $\pi n$ of $\mathscr{A}_{1} \mid \mathscr{C}_{1}^{n}$ if and only if:

$$
\left\{\begin{array}{l}
\left(D_{A}-i * D_{A}\right) \Phi=0 \\
B_{A}=+w .
\end{array}\right.
$$

We shall study this system by the complex variable method of Taubes. Let $z=x_{1}+i x_{2} \in \mathbb{C}, \partial=\frac{\partial}{\partial z}=(1 / 2)\left(\partial_{1}-i \partial_{2}\right), \bar{\partial}=\frac{\partial}{\partial \bar{z}}=(1 / 2)\left(\partial_{1}+i \partial_{2}\right)$ and $\alpha=(i / 2)\left(A_{1}+i A_{2}\right)$. Observe

$$
\nabla_{A}-i * \nabla_{A}=\left(\begin{array}{l}
\partial_{1}+i \partial_{2}-i A_{1}+A_{2} \\
\partial_{2}-i \partial_{1}-i A_{2}-A_{1}
\end{array}\right)=\left(\begin{array}{cc}
2 & 0 \\
0 & -2 i
\end{array}\right)\left(\begin{array}{l}
\bar{\partial}-\alpha \\
\bar{\partial}-\alpha
\end{array}\right) .
$$

On the other hand

$$
B_{A}=i \operatorname{div} A-4 \partial \alpha \text {. }
$$

In conclusion, in order to classify all the configurations $(A, \Phi)$ such that $\mathscr{A}_{1}(A, \Phi)=\pi n$ it is enough to describe all the solutions $\alpha, \Phi$ of the system:

$$
\left\{\begin{array}{l}
(\bar{\partial}-\alpha) \Phi=0 \\
\operatorname{Re} \partial \alpha=-(1 / 4) w \equiv(1 / 8)\left(|\Phi|^{2}-1\right) .
\end{array}\right.
$$

The unknowns are the complex functions $\alpha$ and $\Phi$ on $\Omega$, such that $\alpha, \Phi \in H^{1},|\Phi|=1$ on $\Gamma$ and $G(\Phi \mid \Gamma)=n$. We have denoted $G(f)$ the topological degree of a function $f: \Gamma \rightarrow S^{1}$ of class $H^{1 / 2}(\Gamma)$ defined by Theorem A.3 of the appendix (see also (2.12) and the remarks preceding it). Remark that the condition $\left.B_{A}\right|_{\Gamma}=0$ (see the definition of a $\mathscr{C}_{1}$-regular stationary configuration) is automatically satisfied.

Remark also that the system (4.12) has a very large invariance group: if $\Lambda \in \mathscr{G} \equiv H_{\text {real }}^{2}$ and $\alpha, \Phi$ are solutions of (4.12) (with all the boundary conditions satisfied, in particular with the $n$ given a priori) then the functions $\alpha_{\Lambda} \equiv \alpha+i \bar{\partial} \Lambda$, $\Phi_{\Lambda} \equiv e^{i \Lambda} \Phi$ (obtained after a gauge transformation on $(A, \Phi)$ ) also verify the system (and the same boundary conditions, with the same $n$ ). In particular a general solution of (4.12) cannot be smooth.

The system (4.12) will be solved using Taubes' procedure of factorizing the solution into a holomorphic part and a factor which is different from zero. As we said at the beginning of this section the main difficulties in our case are due to the presence of the boundary $\Gamma$. In order to overcome them, we shall first study 
some properties of holomorphic functions of Sobolev class $H^{1}$ in $\Omega$. The main technical point is part (a) of the proposition below.

Proposition 4.1. (a) Let $F$ be a bounded harmonic function in $\Omega$ of Sobolev class $H^{1}(\Omega)$. Assume that on a neighbourhood in $\Gamma$ of a point $x_{0} \in \Gamma$ we have $\mid F \|_{\Gamma} \geqq$ const. $>0$. Then there is a neighbourhood of $x_{0}$ in $\bar{\Omega}$ on which $|F| \geqq$ const. $>0$.

(b) Let $V=\left\{f \in L^{2}(\Omega) \mid \bar{\partial} f \in L^{2}(\Omega)\right\}$ provided with the norm $\|f\|_{V}=\left(\|f\|^{2}+\|\bar{\partial} f\|^{2}\right)^{1 / 2}$. Then $C^{\infty}(\bar{\Omega})$ is dense in $V$ and the application $\left.C^{\infty}(\bar{\Omega}) \ni f \mapsto f\right|_{\Gamma} \in C^{\infty}(\Gamma)$ has a unique continuous extension to an application of $V$ into $H^{-1 / 2}(\Gamma)$.

(c) Let $f$ be a holomorphic function on $\Omega$ which is bounded and of Sobolev class $H^{1}(\Omega)$. If $|f| \geqq$ const. $>0$ on $\Gamma$, then $f$ has only a finite number $N$ of zeros in $\Omega$ and we have the usual formula:

$$
(1 / 2 \pi i) \oint_{\Gamma} \frac{f^{\prime}(z)}{f(z)} d z=\sum_{k=1}^{N} n_{k},
$$

where $n_{k}$ is the multiplicity of the $k^{\text {th }}$ zero and the integral is interpreted in the distributional sense.

Proof. (a) Since the assertion is purely local, we shall consider a smooth simply connected open set $U$ in $\Omega$ whose boundary contains a neighbourhood of $x_{0}$ in $\Gamma$. Then we map $U$ on the upper half-plane using a conformal transformation. Thus it is enough to prove the following fact: if $\Omega$ is the upper half-plane, $f: \mathbb{R} \rightarrow \mathbb{C}$ is a bounded function which is of class $H^{1 / 2}$ on some open interval $I \subset \mathbb{R}$, then for any $x \in I$ and any neighbourhood $J$ of $x$ in $I$ the distance from $f_{\varepsilon}(x)$ to the image $f(J)$ tends to zero as $\varepsilon \rightarrow 0$, where

$$
f_{\varepsilon}(x)=(\varepsilon / \pi) \int_{\mathbb{R}} f(t)\left((x-t)^{2}+\varepsilon^{2}\right)^{-1} d t
$$

and the connection with our problem is given by the formula $F(x+i y)=f_{y}(x)$, where $F$ is the harmonic extension of $f$ to $\Omega$. Let $P_{\varepsilon}(x) \equiv \varepsilon /\left(\pi\left(x^{2}+\varepsilon^{2}\right)\right)$ be the Poisson kernel for the upper half-plane. For each $a>0$ we define $\lambda>0$ by the equality:

$$
\int_{|x|>\varepsilon \lambda / 2} P_{\varepsilon}(x) d x \equiv 1 / \pi[\pi-2 \operatorname{arctg} \lambda / 2]=a .
$$

Since $P_{\varepsilon}(x) \leqq(\varepsilon \pi)^{-1}$, we have for all $x, y \in \mathbb{R}$ :

$$
\begin{aligned}
\left|f_{\varepsilon}(x)-f(y)\right| & \leqq \int_{\mathbb{R}} P_{\varepsilon}(x-t)|f(t)-f(y)| d t \\
& \leqq(\pi \varepsilon)^{-1} \int_{|x-t| \leqq \varepsilon \lambda / 2}|f(t)-f(y)| d t+\int_{|x-t|>\varepsilon \lambda / 2} P_{\varepsilon}(x-t) d t \cdot 2 \sup _{t \in \mathbb{R}}|f(t)| \\
& =(\pi \varepsilon)^{-1} \int_{|x-t| \leqq \varepsilon \lambda / 2}|f(t)-f(y)| d t+2 a \sup |f| .
\end{aligned}
$$

Thus if we denote

$$
I_{\varepsilon}(x)=(x-\lambda \varepsilon / 2, x+\lambda \varepsilon / 2)
$$

and

$$
M_{\varepsilon}(x)=\left[\iint_{I_{\varepsilon} \times I_{\varepsilon}}|f(y)-f(t)|^{2}|y-t|^{-2} d t d y\right]^{1 / 2},
$$


we have

$$
\begin{aligned}
(\lambda \varepsilon)^{-1} \int_{I_{\varepsilon}}\left|f_{\varepsilon}(x)-f(y)\right| d y & \leqq 2 a \sup |f|+(\lambda / \pi)(\lambda \varepsilon)^{-2} \iint_{I_{\varepsilon} \times I_{\varepsilon}}|f(t)-f(y)| d t d y \\
& \leqq 2 a \sup |f|+(\lambda / \pi)\left[(\lambda \varepsilon)^{-2} \iint_{I_{\varepsilon} \times I_{\varepsilon}}|f(t)-f(y)|^{2} d t d y\right]^{1 / 2} \\
& \leqq 2 a \sup |f|+(\lambda / \pi)\left[\iint_{I_{\varepsilon} \times I_{\varepsilon}}|f(t)-f(y)|^{2}|t-y|^{-2} d t d y\right]^{1 / 2}
\end{aligned}
$$

because $\lambda \varepsilon \geqq|t-y|$ in the domain of integration.

Remark that $M_{\varepsilon}(x) \rightarrow 0$ as $\varepsilon \rightarrow 0$ uniformly for $x$ in compact subsets of $I$, because $f \in H^{1 / 2}(I)$. Now, as in the appendix

$$
\left|\left\{y \in I_{\varepsilon}(x)|| f_{\varepsilon}(x)-f(y) \mid \geqq \beta\right\}\right| \leqq(\lambda \varepsilon / \beta)\left[2 a \sup |f|+(\lambda / \pi) M_{\varepsilon}(x)\right] .
$$

Hence:

$$
\left|\left\{y \in I_{\varepsilon}(x)|| f_{\varepsilon}(x)-f(y) \mid<\beta\right\}\right| \geqq \lambda \varepsilon\left[1-\left(2 a \sup |f|+\lambda \pi^{-1} M_{\varepsilon}(x)\right) \beta^{-1}\right]
$$

which is strictly positive if $2 a \sup |f|+(\lambda / \pi) M_{\varepsilon}(x)<\beta$. This can clearly be achieved for any $\beta>0$ and all $x$ in a compact subset of $I$ by taking first $a$ small (which fixes $\lambda$ ) and then letting $\varepsilon \rightarrow 0$. This proves (a).

(b) The density of $C^{\infty}(\bar{\Omega})$ in $V$ follows from the "weak equal strong" lemma of Friedrichs. Let $v=v_{1}+i v_{2}: \Gamma \rightarrow \mathbb{C}$, which is a $C^{\infty}$ function on $\Gamma$ with $|v(x)| \equiv 1$. An integration by parts yields for $f, g \in H^{1}(\Omega)$ :

$$
\langle g, \bar{\partial} f\rangle+\langle\partial g, f\rangle=(1 / 2)\langle g, v f\rangle_{\Gamma},
$$

where $v f$ is the usual product of complex functions on $\Gamma$. Thus there is a constant $c_{1}$ such that:

$$
\left|\langle g, v f\rangle_{\Gamma}\right| \leqq 2\|g\|\|\bar{\partial} f\|+2\|\partial g\|\|f\| \leqq c_{1}\|g\|_{H^{1}(\Omega)}\|f\|_{V} .
$$

There is a linear continuous application $R: H^{1 / 2}(\Gamma) \rightarrow H^{1}(\Omega)$ such that $\left.(R \varphi)\right|_{\Gamma}=\varphi$ for all $\varphi \in H^{1 / 2}(\Gamma)$, so

$$
\left|\langle\varphi, v f\rangle_{\Gamma}\right| \leqq c_{1}\|R \varphi\|_{H^{1}(\Omega)}\|f\|_{V} \leqq c_{2}\|\varphi\|_{H^{1 / 2}(\Gamma)}\|f\|_{V},
$$

for all $\varphi \in H^{1 / 2}(\Gamma)$. Replacing $\varphi$ by $v \varphi$ and taking into account that $v \in C^{\infty}(\Gamma)$ and $|v(x)| \equiv 1$, we get:

$$
\|f\|_{H^{-1 / 2}(\Gamma)} \leqq c\|f\|_{V}
$$

for all $f \in H^{1}(\Omega)$ and some finite constant $C$. This proves (b).

(c) By (a), $|f|$ will be bounded below by a strictly positive constant on a neighbourhood of the boundary $\Gamma$ in $\Omega$, hence it has only a finite number of zeros in $\Omega$. Clearly $f^{\prime} \in L^{2}(\Omega)$ and is holomorphic, so, by part (b), $f^{\prime} \mid \Gamma$ is a well defined element of $H^{-1 / 2}(\Gamma)$. Also, since $|f| \geqq$ const. $>0$ on $\Gamma$ and $f \in H^{1 / 2}(\Gamma)$, we also have $\left.f^{-1}\right|_{\Gamma} \in H^{1 / 2}(\Gamma)$. We shall interpret the integral appearing in the left-hand side of (4.13) using the anti-duality map $\langle\cdot, \cdot\rangle_{\Gamma}: H^{1 / 2}(\Gamma) \times H^{-1 / 2}(\Gamma) \rightarrow \mathbb{C}$ :

$$
\oint_{\Gamma} \frac{f^{\prime}(z)}{f(z)} d z=\left\langle\bar{f}^{-1}, f^{\prime} \tau\right\rangle_{\Gamma} \equiv\left\langle\bar{f}^{-1}, d f \mid d \sigma\right\rangle_{\Gamma},
$$


where $\tau=\tau_{1}+i \tau_{2}: \Gamma \rightarrow \mathbb{C}$ is a $C^{\infty}$ function and $d / d \sigma$ is the tangential derivative on $\Gamma$ (we have $f^{\prime} \tau=(d / d \sigma) f$ for holomorphic $f$ ). In order to prove (4.13), let $\Gamma_{\varepsilon}=\{z \in \Omega \mid \operatorname{dist}(z, \Gamma)=\varepsilon\}$. For $\varepsilon$ small enough, (4.13) is true with $\Gamma$ replaced by $\Gamma_{\varepsilon}$. If we make $\varepsilon \rightarrow 0$ in the left-hand side we get (4.13) by a simple continuity argument.

Let us now return to the boundary value problem, (4.12). Our first result concerns the zeros of $\Phi$.

Proposition 4.2. Let $\alpha, \Phi \in H^{1}$ satisfy (4.12) with $|\Phi|=1$ on $\Gamma$ and $G\left(\left.\Phi\right|_{\Gamma}\right)=$ $n(n=0,1,2, \ldots)$. Then $\Phi \in H_{\text {loc }}^{2}$ and either $|\Phi(z)|=1$ for all $z \in \Omega$ or $|\Phi(z)|<1$ for all $z \in \Omega$. Moreover, $\Phi$ has only a finite number of zeros $z_{1}, \ldots, z_{k}$ in $\Omega$ and there are integers $n_{1}, \ldots, n_{k} \geqq 1$ such that $n_{1}+\cdots+n_{k}=n$, and a function $\Phi_{0} \in H_{\text {loc }}^{2}(\Omega) \cap H^{1}$ with $\left|\Phi_{0}(z)\right| \geqq$ const. $>0$ on $\Omega$, such that:

$$
\Phi(z)=\prod_{j=1}^{k}\left(z-z_{j}\right)^{n_{j}} \Phi_{0}(z) .
$$

Proof. Let $\rho(z) \equiv(\pi z)^{-1}$ be the fundamental solution of $\bar{\partial}$ in $\mathbb{R}^{2}$, i.e. $\bar{\partial}(\rho \times f)=f$ for $f \in L^{1}\left(\mathbb{R}^{2}\right)$ with compact support (here and below $\times$ denotes the convolution product). We shall use the following known properties: if $f \in L^{q}\left(\mathbb{R}^{2}\right)$ for some $q>2$, then $\rho \times f$ is continuous; and if $f \in H^{1}\left(\mathbb{R}^{2}\right)$ then $\rho \times f \in H_{\text {loc }}^{2}\left(\mathbb{R}^{2}\right)$. Let $\alpha_{0} \in H^{1}\left(\mathbb{R}^{2}\right)$ have compact support and $\left.\alpha_{0}\right|_{\Omega}=\alpha$. We put $b_{0}=\exp \left(\rho \times \alpha_{0}\right)$. It is clear that $b_{0} \in H_{\text {loc }}^{2}\left(\mathbb{R}^{2}\right), \bar{\partial} b_{0}=\alpha_{0} b_{0}$. Moreover $b_{0}^{-1}=\exp \left(-\rho \times \alpha_{0}\right) \in H_{\text {loc }}^{2}\left(\mathbb{R}^{2}\right)$. We put $b=\left.b_{0}\right|_{\Omega}$ and define $h: \Omega \rightarrow \mathbb{C}$ by the condition $\Phi=b h$. It follows that $h$ is a holomorphic function in $\Omega$ of Sobolov class $H^{1}$. Moreover $|h|=\left|b^{-1}\right|$ is a bounded function on $\Gamma$ such that $|h| \geqq$ const. $>0$ (because $b$ is continuous and non-zero). Hence $h \in L^{\infty}(\Omega)$ also. Using part (c) of Proposition 4.1 it follows that $\Phi$ has a finite number of zeros, the same as those of $h$. By an argument similar to that from the end of the proof of Proposition 4.1 (i.e. calculate the degree by replacing $\Gamma$ by $\Gamma_{\varepsilon}$ ) one can easily prove that

$$
G\left(\left.\Phi\right|_{\Gamma}\right)=G\left(\left.b\right|_{\Gamma}\right)+G\left(\left.h\right|_{\Gamma}\right)=G\left(\left.h\right|_{\Gamma}\right),
$$

since $b$ is a pure exponential and is of class $H^{2}$. In order to prove $n=n_{1}+\cdots+n_{k}$, we use Proposition 2.4 and formulas (4.13) and (4.14). Finally, Thecrem 3.2 may be applied because $B_{A}=w=(1 / 2)\left(1-|\Phi|^{2}\right) \in H^{1}$, so that $(A, \Phi)$ is a $\mathscr{C}_{1}$-regular stationary configuration.

In order to give a complete classification of the solutions of (4.12) we make two more remarks. First, if $\psi \in H^{1 / 2}\left(\Gamma_{j}\right)$ and $|\psi| \geqq$ const. $>0$, we define its winding number by:

$$
G(\psi)=(1 / 2 \pi i)\left\langle\bar{\psi}^{-1}, d \psi / d \sigma\right\rangle_{\Gamma_{j}} .
$$

As in Theorem A.3 this is an integer. Secondly, let $\psi \in H^{1}(\Omega) \cap H_{\text {loc }}^{2}(\Omega)$ be such that $|\psi| \geqq$ const. $>0$ and $G\left(\psi \mid \Gamma_{j}\right)=0$ for all $j=0,1, \ldots, m$. Then there is a $\theta \in H^{1}(\Omega) \cap H_{\text {loc }}^{2}(\Omega)$ such that $\underset{m}{\psi}=e^{\theta}$. In fact, let $\Omega_{\varepsilon}=\{z \in \Omega \mid \operatorname{dist}(z, \Gamma)>\varepsilon\}$ with $\varepsilon$ very small. Then $\partial \Omega_{\varepsilon}=\Gamma_{\varepsilon}=\bigcup_{j=0}^{m} \Gamma_{j, \varepsilon}$ and it is easily seen that $G\left(\left.\psi\right|_{\Gamma_{j, \varepsilon}}\right)=G\left(\left.\psi\right|_{\Gamma_{j}}\right)=0$.

Since $\psi$ is continuous on $\Omega_{\varepsilon}$, it is well-known that there is a continuous function $\theta$ on $\Omega_{\varepsilon}$ (unique modulo an additive constant) such that $\psi=e^{\theta}$ on $\Omega_{\varepsilon}$. Making 
$\varepsilon \rightarrow 0$ we extend $\theta$ to a continuous function on $\Omega$ such that the equality remains true. It is easy to show that $\nabla \theta=\psi^{-1} \nabla \psi$. The right-hand side belongs to $L^{2}(\Omega)$ because $\psi^{-1}$ is bounded. So $\theta \in H^{1}(\Omega)$ and the fact that $\theta \in H_{\text {loc }}^{2}(\Omega)$ follows from the same identity.

Let us assume that we are in the conditions of Proposition 4.2 and denote $G\left(\left.\Phi\right|_{\Gamma_{i}}\right)=N_{i} \in \mathbb{Z}, i=0,1, \ldots, m$ (see the end of Sect. 2). Then $G\left(\left.\Phi\right|_{\Gamma}\right) \equiv n=N_{0}+$ $N_{1}+\cdots+N_{m}$. Choose $\zeta_{1}, \ldots, \zeta_{m} m$ points in the bounded domains limited by the curves $\Gamma_{1}, \ldots, \Gamma_{m}$ and define $\psi: \Omega \rightarrow \mathbb{C}$ by

$$
\Phi(z)=\prod_{j=1}^{k}\left(z-z_{j}\right)^{n_{j}} \prod_{i=1}^{m}\left(z-\zeta_{i}\right)^{N_{i}} \psi(z) .
$$

Clearly $\psi \in H^{1} \cap H_{\text {loc }}^{2}(\Omega)$ and $0<$ const. $\leqq|\psi(z)| \leqq$ const. $<\infty$. Moreover $G\left(\left.\psi\right|_{\Gamma_{i}}\right)=0$ for all $i=0,1, \ldots, m$ (use the equality with $G\left(\psi \mid \Gamma_{i, \varepsilon}\right)$ for small $\varepsilon$, see above and the end of the proof of Proposition 4.1). Hence $\psi=e^{\theta}$ with $\theta \in H^{1} \cap H_{\text {loc }}^{2}(\Omega)$. Clearly $\bar{\partial} \psi=\psi \cdot \bar{\partial} \theta$ and the first equation (4.12) implies $\alpha=\bar{\partial} \theta$. The second equation (4.12) becomes $\Delta \operatorname{Re} \theta=-w \equiv(1 / 2)\left(|\Phi|^{2}-1\right)$.

We define $u=\log |\psi|^{2} \equiv 2 \operatorname{Re} \theta$ which is clearly in $H^{1} \cap H_{\text {loc }}^{2}$ and:

$$
a(z)=\prod_{j=1}^{k}\left|z-z_{j}\right|^{2 n_{j}} \prod_{i=1}^{m}\left|z-\zeta_{i}\right|^{2 N_{i}} .
$$

Then $a \in C^{\infty}(\bar{\Omega})$ and $a(z)>0$ on $\Gamma$. Let $\varphi=-\left.\ln a\right|_{\Gamma} \in C^{\infty}(\Gamma)$. Hence $u$ is a solution of the following boundary value problem:

$$
\left\{\begin{array}{l}
-\Delta u+a e^{u}=1 \\
\left.u\right|_{\Gamma}=\varphi
\end{array}\right.
$$

We shall prove later on the following general result:

Theorem 4.3. Let $k \geqq-1$ be an integer. Assume three real functions $a \in H^{k}(\Omega)$, $v \in H^{k}(\Omega), \varphi \in H^{k+3 / 2}(\Gamma)$ are given such that $a \geqq 0$ and $a \in L^{1+\varepsilon}(\Omega)$ for some $\varepsilon>0$. Then there is a unique real function $u \in H^{1}(\Omega)$ such that:

$$
\left\{\begin{array}{l}
-\Delta u+a e^{u}=v \\
\left.u\right|_{\Gamma}=\varphi
\end{array}\right.
$$

Moreover, we have $u \in H^{k+2}(\Omega)$. In particular, if $a, v \in C^{\infty}(\bar{\Omega})$ and $\varphi \in C^{\infty}(\Gamma)$, then $u \in C^{\infty}(\bar{\Omega})$.

We can now describe all the configurations in $\mathscr{C}_{1}^{n}$ on which $\mathscr{A}_{1}$ assumes its absolute minimum $\pi n$.

Theorem 4.4. Let $n \geqq 0$ an integer and $N_{0}, N_{1}, \ldots, N_{m} \in \mathbb{Z}$ such that $N_{0}+N_{1}+\cdots$ $+N_{m}=n$. Let $z_{1}, \ldots, z_{k} \in \Omega$ and $\zeta_{i}$ in the bounded domain limited by $\Gamma_{i}, i=1, \ldots, m$. Finally, let $n_{1}, \ldots, n_{k} \geqq 1$ be integers with $n_{1}+\cdots+n_{k}=n$ (if $n=0$, the set $\left\{z_{1}, \ldots, z_{k}\right\}$ is empty by definition). Let $u$ be the unique solution of (4.18) with a and $\varphi$ defined as above. Then

$$
\left\{\begin{array}{l}
\Phi(z)=\prod_{j=1}^{k}\left(z-z_{j}\right)^{n_{j}} \prod_{i=1}^{m}\left(z-\zeta_{i}\right)^{N_{i}} e^{u(z) / 2} \\
A(z)=(-1 / 2) * \nabla u(z)
\end{array}\right.
$$


defines a configuration $(A, \Phi) \in \mathscr{C}_{1}^{N_{0} \cdots N_{m}}$ such that $\mathscr{A}_{1}(A, \Phi)=\pi n$. Moreover, any configuration in $\mathscr{C}_{1}^{N_{0} \cdots N_{m}}$ on which $\mathscr{A}_{1}$ assumes its absolute minimum $\pi n$ is gauge equivalent to one of those described above. In particular, any such configuration has the property $\Phi \in H^{2},|\Phi|^{2} \in C^{\infty}(\bar{\Omega})$ and $B_{A} \in C^{\infty}(\bar{\Omega})$ and is gauge-equivalent to a configuration with $C^{\infty}(\bar{\Omega})$ components.

Remarks. The points $\zeta_{i}$ are not determined by the function $\Phi$; they may be chosen a priori and considered as fixed. This follows from the construction of $\psi$ in (4.17). Solutions (4.20) with $N_{1}=\cdots=N_{m}=0$ are called of multi-vortex type. The other ones are of mixed vortex-antivortex type in general (if some $N_{i}$ are negative) and do not appear in the case of simply connected regions. If $n<0$, one must replace in the preceding analysis "holomorphic" by "antiholomorphic" and $z-z_{j}, z-\zeta_{i}$ by $\bar{z}-\bar{z}_{j}, \bar{z}-\bar{\zeta}_{j}$.

Theorem 4.4 follows easily from Theorem 4.3 and the construction we have done before. We only want to observe that $\theta$ is of Sobolev class $H^{2}$. In fact, $\alpha=\bar{\partial} \theta \in H^{1}$ and $\operatorname{Re} \theta=u / 2 \in C^{\infty}(\bar{\Omega})$ so $\bar{\partial} \operatorname{Im} \theta \in H^{1} ; \operatorname{Im} \theta$ being real, this implies $\operatorname{Im} \theta \in H^{2}$. Making a gauge-transformation $\Lambda=-\operatorname{Im} \theta$, we may suppose $\theta=\operatorname{Re} \theta=u / 2$, which gives the unique solution (4.20).

We finish by giving the proof of Theorem 4.3. From now on we shall work only with real functions, so we simplify the notation: $H^{s, p} \equiv H_{\text {real }}^{s, p}, L^{p} \equiv L_{\text {real }}^{p}$, etc. We shall use the following inequality (see [7] p. 155): there is a constant $c$ depending only on $\Omega$ such that for all $u \in H^{1}(\Omega)$,

$$
\int_{\Omega} \exp \left[c^{-1}\|u\|_{H^{1}}^{-2}|u|^{2}\right] d x \leqq c
$$

(because we can extend $u$ to an element $\tilde{u} \in H_{0}^{1}(\tilde{\Omega})$ for some fixed $\tilde{\Omega}$ open, bounded, with $\bar{\Omega} \subset \tilde{\Omega}$ ). Then taking $\varepsilon=2 c^{-1}\|u\|_{H^{1}}^{-2}$ we get for any $p<\infty$ :

$$
p|u| \leqq \frac{\varepsilon}{2}|u|^{2}+\frac{1}{2 \varepsilon} p^{2}=c^{-1}\|u\|_{H^{1}}^{-2}|u|^{2}+\frac{c p^{2}}{4}\|u\|_{H^{1}}^{2} .
$$

In conclusion, we get:

$$
\left\|e^{|u|}\right\|_{L^{p}(\Omega)} \leqq c^{1 / p} \exp \left[c p\|u\|_{H^{1}(\Omega)}^{2}\right]
$$

with the same constant as before, all $p<\infty$ and all $u \in H^{1}(\Omega)$. In particular, the application exp: $u \mapsto e^{u}$ sends $H^{1}$ into $\bigcap_{p<\infty} L^{p}$. Moreover, for each $p$, exp: $H^{1} \rightarrow L^{p}$ is continuous and even continuously Frechet derivable, its derivative at the point $u \in H^{1}$ being the linear continuous application: $H^{1} \ni v \mapsto e^{u} v \in L^{p}$. In order to prove this we use the inequality $\left|e^{x}-1-x\right| \leqq|x|^{2} e^{|x|}$ valid for all real $x$ and denote by the same letter $c$ all the irrelevant constants. We get:

$$
\begin{aligned}
& \left\|e^{u+v}-e^{u}-e^{u} v\right\|_{L^{p}}=\left\|e^{u}\left(e^{v}-1-v\right)\right\|_{L^{p}} \\
& \quad \leqq\left\|e^{u} v^{2} e^{|v|}\right\|_{L^{p}} \leqq\left\|e^{u}\right\|_{L^{4 p}}\|v\|_{L^{4 p}}^{2}\left\|e^{|v|}\right\|_{L^{4 p}} \\
& \quad \leqq c\|v\|_{H^{1}}^{2} \exp \left(c\|u\|_{H^{1}}^{2}+c\|v\|_{H^{1}}^{2}\right) .
\end{aligned}
$$

Since $L^{p} \subset H^{-1}$ if $p>1$, it follows that exp considered as an application from $H^{1}$ to $H^{-1}$ is also continuously Frechet derivable.

Let us define $T: H^{1}(\Omega) \rightarrow H^{-1}(\Omega)$ by $T(u)=-\Delta u+a e^{u}$. Since $a \in L^{p}$ for some 
$p>1$, it is easy to see that $T$ is well defined, continuous and even continuously Frechet derivable.

Let $T_{0}=T \mid H_{0}^{1}(\Omega)$. If $u, v \in H_{0}^{1}$ :

$$
\begin{aligned}
\left\langle u-v, T_{0}(u)-T_{0}(v)\right\rangle & =\|\nabla(u-v)\|^{2}+\int_{\Omega} a\left(e^{u}-e^{v}\right)(u-v) d x \\
& \geqq\|\nabla(u-v)\|^{2} \geqq c\|u-v\|_{H^{1}}^{2},
\end{aligned}
$$

where we have used the assumption $a \geqq 0$, the fact that $\left(e^{x}-e^{y}\right)(x-y) \geqq 0$ and the boundedness of $\Omega$. Here $c>0$ is a constant. The above inequality and a well known theorem of F. Browder (see [1] for example) implies that $T_{0}: H_{0}^{1}(\Omega) \rightarrow H^{-1}(\Omega)$ is a homeomorphism.

Remark. The result of Browder we use is a non-linear variant of the Lax-Milgram lemma. More precisely, it says that if $X$ is a real Hilbert space, $X^{*}$ its dual and $F: X \rightarrow X^{*}$ is continuous and has the property: $\langle x-y, F(x)-F(y)\rangle \geqq c\|x-y\|_{X}^{2}$ for some constant $c>0$ and all $x, y \in X$, then $F$ is a homeomorphism of $X$ onto $X^{*}$. We use this result only for a continuously Frechet derivable $F$ and in this case the proof is very easy. We give it for completeness. First, from $c\|x-y\|_{X}^{2} \leqq\langle x-y, F(x)-F(y)\rangle \leqq$ $\|x-y\|_{X}\|F(x)-F(y)\|_{X^{*}}$ one gets $\|F(x)-F(y)\|_{X^{*}} \geqq c\|x-y\|_{X}$ so $F$ is injective, with closed range $F(X)=Y \subset X^{*}$ and continuous inverse $F^{-1}: Y \rightarrow X$ (even Lipschitz). We have to show that $Y=X^{*}$. But it is clear that the derivative $F^{\prime}\left(x_{0}\right): X \rightarrow X^{*}$ at any point $x_{0}$ will have the property $c\|v\|_{X}^{2} \leqq\left\langle v, F^{\prime}\left(x_{0}\right) v\right\rangle$. Now the linear variant of the Lax-Milgram lemma implies that $F^{\prime}\left(x_{0}\right)$ is a linear homeomorphism of $X$ onto $X^{*}$. The inverse mapping theorem assures us that $F$ has open image, so $Y=X^{*}$.

The preceding arguments show the existence and unicity of the solution in the case $\varphi=0$. In order to prove uniqueness in general, consider $u_{1}, u_{2} \in H^{1}$ solutions of (4.19) and denote $u=u_{1}-u_{2} \in H_{0}^{1}, b=a e^{u_{2}}$. Since $a \in L^{p}$ for some $p>1$ and $e^{u_{2}} \in L^{q}$ for all $q<\infty$, we have $b \geqq 0$ and $b \in L^{p}$ for some $p>1$. Clearly we have $-\Delta u+b\left(e^{u}-1\right)=0$. Let $S: H_{0}^{1} \rightarrow H^{-1}$ be given by $S(u)=-\Delta u+b\left(e^{u}-1\right)$. Exactly as before we see that $S$ is well defined (even continuously Frechet derivable) and $\langle u-v, S(u)-S(v)\rangle \geqq c\|u-v\|_{H^{1}}^{2}$, hence $S$ is injective. This implies $u=0$, i.e. we have uniqueness of the solution of (4.19). To prove the existence let $u_{0} \in H^{k+2}$ with $u_{0} \mid \Gamma=\varphi$ and look for $u$ of the form $u=w+u_{0}$ with $w \in H_{0}^{1}$. The $w$ must satisfy $-\Delta w+a e^{u_{0}} e^{w}=v+\Delta u_{0}$. Since $k+2 \geqq 1$, we have $e^{u_{0}} \in L^{q}$ for all $q<\infty$, so $a e^{u_{0}} \in L^{p}$ for some $p>1$ and is positive. Also $v+\Delta u_{0} \in H^{k}$ and $k \geqq-1$. Hence the existence of a solution $w \in H_{0}^{1}$ follows from the properties of $T$ (in which $a$ is replaced by $\left.a e^{u_{0}}\right)$.

We have proved the existence and uniqueness of the solution $u \in H^{1}$ of the system (4.19). We must prove now that $u \in H^{k+2}$. Of course, we can assume $k \geqq 0$. First we make a reduction to the case $\varphi=0$ introducing a function $u_{0}$ as before. Then $v+\Delta u_{0} \in H^{k}$ is trivial and $a e^{u_{0}} \in H^{k}$ is proved by induction on $k$ (if $k=0$, then $u_{0} \in C^{0}(\bar{\Omega})$, hence the assertion is trivial; if $k=1: \nabla\left(a e^{u_{0}}\right)=\nabla a \cdot e^{u_{0}}+a \nabla u_{0} \cdot e^{u_{0}} \in H^{0}$, so $a e^{u_{0}} \in H^{1}$, etc...). So, we may consider $\varphi=0$ and $k \geqq 0 . u \in H_{0}^{1}$ has the property $\Delta u=a e^{u}-v$ and $a, v \in H^{k}$. Then $a e^{u} \in L^{p}$ for all $p<2$. Theorem 8.16 of [7] implies $u \in L^{\infty}$. But then $a e^{u}-v \in L^{2}$, so $u \in H^{2}$ due to $H^{s}$-regularity for the Dirichlet problem. In particular $u \in C^{0}(\bar{\Omega})$. If $k \geqq 1$, then $\nabla\left(a e^{u}\right)=\nabla a \cdot e^{u}+a \nabla u e^{u} \in L^{2}$ because $a$ and $\nabla u$ are of class $H^{1}$, hence are in $L^{p}$ for all $p<\infty$. So $a e^{u} \in H^{1}$ which implies $\Delta u \in H^{1}$. 
Since $u \in H_{0}^{1}$, we get $u \in H^{3}$. If $k \geqq 2$ we shall use two facts: 1) $H^{k}$ is an algebra for the usual multiplication of functions; 2) if $u \in H^{k+1}$ then $e^{u} \in H^{k}$. The first is well-known and easily proved by induction. The second is a consequence of the first because it is enough to show $\nabla e^{u} \equiv \nabla u \cdot e^{u} \in H^{k-1}$ which is clear by induction. In conclusion, if $u \in H^{k+1}$ and $a \in H^{k}$, then $a e^{u} \in H^{k}$. The proof of Theorem 4.4 is finished by a new induction procedure.

\section{Appendix}

As mentioned in the introduction the result of this appendix is due to L. Boutet de Monvel, and for one crucial point, to O. Gabber. It was exposed orally in a lecture by L. Boutet de Monvel in the Franco-Israelian Congress on P.D.E.'s in Jerusalem, April 1984. We thank them for providing us with this material.

$A .1$. Let $S^{1}$ denote the unit circle in the complex plane $\mathbb{C}$. With its geodesic measure $S^{1}$ is also identified with $\mathbb{R} / 2 \pi \mathbb{Z}$. We will say that a measurable function $f$ from $S^{1}$ to $\mathbb{R}^{n}$ belongs to VMO, the space of functions with vanishing mean oscillation, if the following condition holds: For any interval $I \subset S^{1}$ we denote $|I|$ the length of $I$, and $f_{I}=\frac{1}{|I|} \int_{I} f$ the mean
of $f$ over $I$; then $f \in$ VMO if

$$
N_{I}(f)=\frac{1}{|I|} \int_{I}\left|f(x)-f_{I}\right| d x \rightarrow 0 \quad \text { if } \quad|I| \rightarrow 0 .
$$

(The integral is taken with respect to the Lebesgue measure of $S^{1}$, for which the total length is $2 \pi$.)

We will set

$$
w(\varepsilon)=\sup _{|I| \leqq \varepsilon} N_{I}(f), \text { thus } \quad \lim _{\varepsilon \rightarrow 0} w(\varepsilon)=0 \quad \text { if } f \in \mathrm{VMO} .
$$

If $f$ is a locally integrable function we set

$$
F(x, \varepsilon)=f_{\varepsilon}(x)=\frac{1}{\varepsilon} \int_{0}^{\varepsilon} f(x+t) d t .
$$

Let us notice that for any interval $I$ and any number $c>0$, the set $I_{c} \subset I$ of points $y \in I$ such that $\left|f(y)-f_{I}\right|>c$ is of length $\left|I_{c}\right| \leqq \frac{1}{c} N_{I}(f)|I|$, since $|I| N_{I}(f) \geqq \int_{I_{\mathrm{c}}}\left|f-f_{I}\right| \geqq c\left|I_{c}\right|$. From this we get

Proposition A.1. If $f \in \mathrm{VMO}$ the distance of $f_{\varepsilon}(x)$ to the range of $f$ is $\leqq w(\varepsilon)$, for any $x \in S^{1}$ and $\varepsilon>0$.

Indeed we have $f_{\varepsilon}(x)=f_{I}$ with $I=[x, x+\varepsilon]$, and if $c>\varepsilon$ the set of $y \in I$ such that $\left|f(y)-f_{I}\right| \leqq c$ is of length $\geqq \varepsilon\left(1-\frac{w(\varepsilon)}{c}\right)>0$, hence non-empty.

If we now suppose that $f$ takes its values in a smooth submanifold $M \subset \mathbb{R}^{n}$, e.g. the circle $S^{1} \subset \mathbb{C}$, it follows from Proposition A.1 that for small $\varepsilon, F(x, \varepsilon)$ takes its 
values in a small fixed tubular neighbourhood of $M$ (which is of course homotopic to $M$ ). Hence $f_{\varepsilon}$ defines a constant (independent of $\varepsilon$ ) homotopy class of $M$, and we can take this as a definition of the homotopy class $[f] \in \pi_{1}(M)$, although $f$ may be discontinuous ( $F$ is of course continuous if $\varepsilon>0$ ).

$A .2$. From now on we specialize to the case where $f$ belongs to the Sobolev space $H^{1 / 2}$, which is the useful case for us.

Proposition A.2. We have $H^{1 / 2} \subset \mathrm{VMO}$.

Proof. If $f \in H^{1 / 2}$ we have

$$
\iint_{S^{1} \times S^{1}}\left|\frac{f(x)-f(y)}{x-y}\right|^{2} d x d y<\infty .
$$

Equivalently

$$
\iint_{S^{1} \times \mathbb{R}}\left|\frac{f(x+\varepsilon)-f(x)}{\varepsilon}\right|^{2} d x d \varepsilon<\infty
$$

(where we have, in the notation identified $S^{1}$ and $\mathbb{R} / 2 \pi \mathbb{Z}$ ).

Now for any interval $I \subset S^{1}$ we have, by Schwarz' inequality

$$
\left|f(x)-f_{I}\right|=\frac{1}{|I|}\left|\int_{I}(f(x)-f(y)) d y\right| \leqq\left(\frac{1}{|I|} \int|f(x)-f(y)|^{2} d y\right)^{1 / 2},
$$

hence also

$$
\begin{aligned}
N_{I}(f) & =\frac{1}{|I|} \int_{I}\left|f-f_{I}\right| \leqq\left(\frac{1}{|I|} \int\left|f-f_{I}\right|^{2}\right)^{1 / 2} \\
& \leqq\left(\frac{1}{|I|^{2}} \iint_{I \times I}|f(x)-f(y)|^{2} d x d y\right)^{1 / 2} \\
& \leqq\left(\int_{|x-y|<|I|}\left|\frac{f(x)-f(y)}{x-y}\right|^{2} d x d y\right)^{1 / 2} .
\end{aligned}
$$

The last inequality holds because $|x-y|<|I|$ if $x, y \in I$. Since integral (A.4) is finite, the last integral tends to 0 if $|I| \rightarrow 0$, so $f$ satisfies (A.1).

We will now prove the following theorem:

Theorem A.3. Let $f$ be a function of Sobolev class $H^{1 / 2}$ from the circle $S^{1}$ to itself. Then there exists an integer $n$ and a real function $g \in H^{1 / 2}$ on $S^{1}$, unique up to an integral multiple of $2 \pi$, such that $f=z^{n} \exp i g$. The winding number $n$ is given by $n=\frac{1}{2 \pi i} \int_{S^{1}} f^{-1} \frac{\partial f}{\partial x} d x$

(The integral is defined in the distribution sense since $f^{-1}=\bar{f} \in H^{1 / 2}$ and $\frac{\partial f}{\partial x} \in H^{-1 / 2}$.) 
It also follows that smooth functions from $S^{1}$ to itself are dense in $H^{1 / 2}$ such functions, for the $\mathrm{H}^{1 / 2}$ topology (one may approximate $g$ by smooth real functions).

Proof. Let us first notice that the function $F(x, \varepsilon)=\frac{1}{\varepsilon} \int_{x}^{x+\varepsilon} f(t) d t$ introduced above belongs to the Sobolev space $H^{1}$. Indeed we have $\frac{\partial F}{\partial x}=\frac{1}{\varepsilon}(f(x+\varepsilon)-f(x))$, and this belongs to $L^{2}$ by (A4)bis. On the other hand we have

$$
\frac{\partial F}{\partial x}-\frac{\partial F}{\partial \varepsilon}=\frac{1}{\varepsilon^{2}} \int_{0}^{\varepsilon}(f(x+t)-f(x)) d t=\int_{0}^{1} s \frac{\partial F}{\partial x}(x, s \varepsilon) d s .
$$

Hence

$$
\left\|\frac{\partial F}{\partial x}-\frac{\partial F}{\partial \varepsilon}\right\|_{L^{2}} \leqq \int_{0}^{1} s\left\|\frac{\partial F}{\partial x}(x, s \varepsilon)\right\|_{L^{2}} d s=\frac{2}{3}\left\|\frac{\partial F}{\partial x}\right\|_{L^{2}},
$$

since

$$
\left\|\frac{\partial F}{\partial x}(x, s \varepsilon)\right\|_{L^{2}}=\frac{1}{\sqrt{s}}\left\|\frac{\partial F}{\partial x}\right\|_{L^{2}}
$$

This being so let us set

$$
n=\frac{1}{2 \pi i} \int_{S^{1}} f^{-1} \frac{\partial f}{\partial x} d x=\frac{1}{2 \pi i} \int_{S^{1}} f_{\varepsilon}^{-1} \frac{\partial f_{\varepsilon}}{\partial x} d x \quad \text { (for small } \varepsilon \text { ). }
$$

The function $F$ is continuous, does not vanish for small $\varepsilon(|F| \geqq 1-w(\varepsilon))$, and its winding number is $n$. It follows that the $\operatorname{logarithm} G=\frac{1}{i} \log z^{-n} F$ is well defined (up to an integral multiple of $2 \pi$ ), and continuous. In fact we have $G \in H^{1}$ since $d G=F^{-1} d F$ has $L^{2}$ coefficients (for small $\varepsilon$ ). Letting $\varepsilon \rightarrow 0$ we get

$$
f=z^{n} \exp i g
$$

with $g$ the boundary value of $G$. We have $g \in H^{1 / 2}$ since $G \in H^{1}$, and $g$ is real (a.e.) since $|f|=1$.

We finally prove the uniqueness statement in the theorem. It is enough to prove that if $g \in H^{1 / 2}$ and expig $=1$, then $g$ is constant. If this were not so, since the values of $g$ are of the form $k 2 \pi$ with $k \in \mathbb{Z}$, there would exist an integer $m$ such that both sets $A=\{g \leqq 2 \pi m\}$ and $B=\{g \geqq m+1\}$ have no zero measure. Let $h=\inf \left(\left(\frac{g}{2 \pi}-m\right)_{+}, 1\right)$; then $h \in H^{1 / 2}$ and $h$ only takes the values 0 (on $A$ ) and 1 (on $B$ ). It follows from the Lebesgue density theorem that there exist arbitrarily small intervals $I$ such that $h_{I}$ is close to 0 , or to 1 . Since $h_{I}$ depends continuously on $I$, there also exist arbitrarily small intervals $I$ such that $h_{I}=1 / 2$. Since $h \in$ VMO (Proposition A2), this contradicts Proposition A1. This ends the proof.

Acknowledgements. We would like to thank Louis Boutet de Monvel not only for communicating to us the material contained in the Appendix, but also for discussions which allowed us to prove boundary regularity in Chapter 4 . 


\section{References}

1. Berger, M.: Nonlinearity and functional analysis. New York: Academic Press 1977

2. Bogomol'nyi, E. B.: J. Iardernoi Fiz. 24, 449 (1976)

3. Deny, J., Lions, J.-L.: Ann. Inst. Fourier V, 305-370 (1953)

4. Georgescu, V.: Ann. Mat. Pura. Appl. 122, 159-198 (1979)

5. Georgescu, V.: Arch. Rat. Mech. Anal. 74, 143-164 (1980)

6. Ghinzburg, V. I., Landau, L. D.: J. Exp. i Teoret. Fiz. 20, (12), (1950)

7. Gilbarg, D., Trudinger, N. S.: Elliptic partial differential equations. Berlin, Heidelberg, New York: Springer 1977

8 Jaffe, A., Taubes, C.: Vortices and monopoles: Structure of static gauge theories. Boston: Birkhaüser 1980

9. Lions, J.-L., Magenes, E.: Ann. Sc. Norm. Sup. Pisca 16, 1-44 (1962)

10. Lions, J.-L., Magenes, E.: Non-homogeneous boundary value problems and applications, I and II. Berlin, Heidelberg, New York: Springer 1972

11. Morrey, Ch.: Multiple integrals in the calculus of variations. Berlin, Heidelberg, New York: Springer 1966

12. Rose-Innes, A.C., Rhoderich, E.N.: Introduction to superconductivity. London: Pergamon Press 1978

13. Saint-James, D., Sarma, G., Thomas, E. J.: Type II superconductivity. London: Pergamon Press 1969

14. Schrieffer, J. R.: Theory of superconductivity New York, Amsterdam: Benjamin 1964

15. Stein, E.: Singular Integrals and differentiability Properties of functions. Princeton: Princeton University Press, 1970

16. Boutet de Monvel, A., Georgescu, V., Purice, R.: Sur un problème aux limites de la théorie de Ginzburg-Landau. C. R. Acad. Sci. Paris 307, série I, 55-58 (1988)

Communicated by H. Araki 
